\title{
Tourist Market Segmentation by Motivation to Shop: A Case Study of Istanbul, Turkey
}

\author{
István Egresi ${ }^{A B}$ \\ Received: March 8, 2017 | Revised: September 21, 2017 | Accepted: October 13, 2017
}

DOI: $10.5937 / g p 21-16078$

\begin{abstract}
Previous research has indicated that shopping could make up for a significant part of the tourist experience and could provide significant benefits to destinations by contributing to local retail revenue and by generating many jobs. In order to design better marketing strategies, destination managers must understand what attracts tourists to a destination and makes them shop while there. However, tourists represent a heterogeneous group and subgroups of individuals are motivated to visit a destination for a variety of reasons. The primary purpose of this study is to segment tourist shoppers visiting Istanbul according to their motivation to shop. Five distinctive groups of 'product-focused shoppers', 'shoppers for cultural experience', 'reluctant shoppers', 'difference seekers' and 'total shoppers' were found and compared by the geographical origin of the tourists, their socio-demographic characteristics, travel characteristics and behaviour, primary motivation for the trip, activity participation and shopping preferences and attitudes. The findings indicate that destination marketers must develop their strategies and marketing products to address the heterogeneity of motivations underlying tourist shopping.
\end{abstract}

Keywords: shopping tourism, shopping motivation, market segmentation, Istanbul, Turkey

\section{Introduction}

Shopping makes up for a significant part of the tourist experience and, in some cases, could also constitute the main motivating factor for travel (Murphy, et al., 2011; Timothy, 2005). Shopping provides benefits to both tourists and destinations (Carmichael, Smith, 2005). On the one hand, for tourists, besides providing them with the things they lack and want, shopping is a means to relax, meet new people and get to know the local culture. On the other hand, at destinations, tourist spending could contribute in a significant way to retail revenue and could generate a significant number of jobs within the local economy (Timothy, 2005). Therefore, it is no surprise that many global cities, such as Dubai (Peter, Anandkumar, 2011) or Singapore (Henderson, et al., 2011), market themselves as shopping destinations. In these cities, shopping tourism is featured in official marketing and development strategies and is promoted as an important element of local economic development (Henderson et al., 2011).

Knowing why people decide to shop when they travel is very important (Bansal, Eiselt, 2004) not least because motivation may be connected with travellers' satisfaction with the trip and the destination (Kozak, 2002; Devesa, et al., 2010). Tourism and destination managers should understand what drives tourists to travel to a destination in order to elevate tourists' satisfaction level and, thus, to increase re-visitation and positive recommendation levels (Yoon, Uysal, 2005).

However, tourists represent a heterogeneous group. Within any tourist group there are subgroups of tourists that are attracted to a destination for different rea-

\footnotetext{
A Department for Management of Science and Technology Development, Ton Duc Thang University, Ho Chi Minh City, Vietnam

B Faculty of Environment and Labour Safety, Ton Duc Thang University, Ho Chi Minh City, Vietnam;

e-mail: istvan.egresi@tdt.edu.vn
} 
sons (Andreu, et al., 2005). Market segmentation refers to a technique used in marketing studies to divide a heterogeneous market into more homogeneous subgroups (Middleton, Clarke, 2001). A number of studies in the past attempted to profile tourist motivations and connect them to their destination choice (Andreu, et al., 2005; Bansal, Eiselt, 2004; Kozak, 2002; among many others). However, segmentation techniques have been rarely employed in 'special interest' tourism markets (Dong, et al., 2013; Kim, Ritchie, 2012) and those studies dedicated specifically to shopping tourism remain particularly limited in number (Choi, et al., 2016).

Moreover, a recent article reviewing the extant literature on shopping tourism (Choi, et al., 2016) found that more than $70 \%$ of all published research papers on shopping tourism are from the USA and East and Southeast Asia while very little has been written about shopping tourism in other parts of the world, including Turkey. This is surprising because Turkey, a country situated between Europe and the Middle East, is a very well-known shopping destination (Egresi, 2017; Egresi, Polat, 2016; Kozak, 2002; Tosun et al., 2007; Yuksel, 2004; 2007).

Attempting to fill this gap, the present study intends to segment the group of visitors to Istanbul into smaller and more homogeneous subgroups based on their motivation to shop while visiting the city. The subgroups will then be profiled and compared based on the geographical origin of the tourists, their sociodemographic characteristics, travel characteristics and behaviour, motivation for the trip, activity participation and shopping preferences and attitudes. Next, the paper will examine how the findings of this study compare to previous research results and will discuss the practical implications of the results. Finally, the limitations of present research will be acknowledged and future research directions will be proposed.

Before discussing the methods used in this study, the paper will proceed with a comprehensive review of the extant literature on shopping tourism and on the motivation to shop while travelling with a special focus on segmentation of shopping tourism markets.

\section{Literature Review}

\section{Motivation to Shop in Shopping Tourism}

Motivation is an important topic in tourism because motives are the starting point that launch decision processes (Fodness, 1994). According to Iso-Ahola (1980), 'a motive is an internal factor that arouses, directs and integrates a person's behaviour'. Motivation arouses from a state of tension and disequilibrium within individuals generated by their internal psychological factors (needs and wants). The decision to trav- el is taken as a counter-action to restore equilibrium through satisfying those needs and wants.

Literature on tourism motivation is dominated by three major theories which explain why people travel, why they select a certain destination and why they engage in certain activities while travelling:

1. Maslow's (1943) need hierarchy theory. According to him, an individual's needs could be classified hierarchically on five levels. The most basic needs refer to access to water, food, rest and shelter. Once these are fulfilled, the individual's needs would move to the next level of the hierarchy until reaching the top level which is self-actualization or personal fulfilment. This is what may prompt travelling and engaging in certain activities at the destination.

2. The push and pull theory (Dann, 1981) according to which people travel motivated by push factors (e.g. their emotional needs) and pull factors (representing the attractive characteristics of a destination). Later, it was determined that push factors will not only determine why and when a person chooses to travel but also where he/she will travel (Crompton, 1979). In other words, if a person is determined to travel by his/her need to shop, he/she will choose a destination that is well-known for its shopping attractions.

3. The seeking and escaping theory (Iso-Ahola, 1982) which established that people engage in tourism to escape their stressful or boring lives and to seek personal rewards and relaxation. Each factor has two dimensions: personal (psychological) and interpersonal (social) (Iso-Ahola, 1990). In other words, a person can engage in tourism activities for cultural enrichment (psychological dimension) and for socialization (social dimension) at the same time.

Numerous motivations were reported by the literature for shopping while travelling. These can be synthesized as follows (Moscardo, 2004; Timothy, 2005; Kim, et al., 2011):

1. To purchase different, unique products that cannot be found or are rare elsewhere;

2. To capitalize on lower prices or on favourable exchange rates;

3. To purchase gifts for friends and relatives in order to strengthen family and social ties;

4. To purchase souvenirs in order to preserve the memory of the places visited;

5. To escape from routine; and

6. To experience a different culture.

According to Keown (1989), relative prices are one of the most influential factors in shopping tourism. He hypothesized that the cheaper the prices in a des- 
tination, the more popular the destination would be for shopping. To the list above, Butler (1991) also added self-esteem, prestige, vanity and nostalgia as valid motivations driving tourists to shop. It is also evident from this list that tourists do not actually need to buy something for shopping to provide them with a pleasurable experience (Choi, et al., 2016; Yuksel, 2004) as many tourists consider shopping at the destination as a leisure and hedonistic activity (Arnold, Reynolds, 2003) or as a way to interact with local people and experience the culture (Choi, et al., 2016). Therefore, Eastlick and Feinberg (1999) classified shopping motives into functional and non-functional. By functional motives they understand tangible attributes such as convenience, variety and quality of the goods sold and of the shop. Non-functional motives refer to non-tangible attributes of the retailer such as company reputation and customers' perception as well as shoppers' need to interact with other people.

Previous research has consistently found that tourists' shopping motivations could also be influenced by the characteristics of the destination and by the nationality and socio-demographic characteristics of tourists. These two factors will be discussed in the next two subsections.

\section{Location as a factor in tourists' motivation to shop}

Location plays an important role in the motivation of tourists to shop while visiting the destination. Some destinations, such as Hong Kong, Singapore, Dubai, New York, London, Paris or Milan are globally or regionally known for their shopping opportunities. For many tourists, shopping is a primary or very important motivation to travel there.

Destination characteristics were found to be another important determinant of tourists shopping motivation and behavior. Some destinations provide tourists with a unique environment (very different from the home environment) which may be stimulating for shopping. Therefore, settings, range of goods and the ambience of the stores could be very important in stimulating tourists to shop (Oh, et al 2004; JansenVerbeke, 1990; Timothy, Butler, 1995).

This unique shopping environment is sometimes part of the local culture and we already know that tourists often engage in shopping at the destination because this activity gives them an opportunity to interact with local people and experience the culture (Choi, et al., 2016; Hsieh, Chang, 2006). For example, in Cappadocia, where shopping was found to be the third most important reason for tourists to visit the region, product authenticity was the most important motivator for tourists to shop there (Tosun, et al., 2007). Therefore, Tosun et al. (2007) suggested promoting shopping as part of the local cultural ex- perience since the main motivation of tourists visiting the region was to experience new cultures and places. With this marketing strategy, more tourists could be attracted to a destination and persuaded to engage in shopping.

Istanbul, the biggest city in Europe and in the Middle East, is not only a major destination for historical and cultural tourism but also an attraction for shopping lovers (Egresi, Arslan, 2016). With over 100 modern shopping centers (one-third of all shopping malls in Turkey) and numerous traditional markets, Istanbul is a major point of attraction for tourist shoppers from all over the world (Egresi, 2015). Istanbul welcomes more than 11 million tourists each year and previous studies have shown that while relatively few of them come to the city specifically to shop, most will do some shopping while there (Egresi, Arslan, 2016).

\section{Nationality and socio-demographic characteristics of tourists}

However, motivations may differ based not only on the characteristics of the destinations but also on the nationality of tourists (Kozak, 2002; Pizam, Sussman, 1995). In general, East Asian tourists were found to be more shopping-oriented than their European or North-American counter-parts (Rosenbaum, Spears, 2005). For example, souvenir and gift shopping was found to be very important for tourists in many parts of the world (Anderson, Littrell 1995; Heung, Cheng, 2000; Timothy, 2005); however, for Asian tourists (Japanese, Taiwanese, etc.) this practice was found to be particularly important in strengthening social relationships (Hobson, Christensen 2001; Kim, et al., 2011; Lin, Lin 2006; Mok, Iverson, 2000).

Many Asians also like to buy luxury items when traveling away from home (Park, et al., 2010) and tend to spend more money on shopping than tourists from other parts of the world. Heung and Qu (1998) have shown that tourists visiting Hong Kong from different countries display different shopping preferences and behaviors. While Chinese and Taiwanese tourists dedicate more than $60 \%$ of their budget to shopping, North-American and European tourists use only about $30 \%$ of their budget for this purpose. Another study found that Europeans visiting a renowned market in Auckland, New Zealand were interested mainly in value for money when buying something and were concerned over "authenticity", whereas Asian shoppers valued more the overall ambience (Kikuchi, Ryan, 2007).

Research has confirmed that not only cultural but also social and demographic characteristics of tourists may influence their motivation to shop while travelling as well as their expectations and preferences (Anderson, Littrell, 1995; Littrell et al., 2004). For example, 
younger and less educated tourists were documented to be more interested in shopping while travelling (Lyu, Noh, 2016), results that can be seen as a major trend shift from previous findings that contended that older tourists with higher education tended to spend more on shopping while on vacation (Mok, Iverson, 2000).In terms of gender, previous studies have found that women, in general, are more interested in shopping and tend to spend more than men (Anderson, Littrell, 1995; Guiry, et al., 2006; Jansen-Verbeke, 1998; Murphy, et al., 2011; Oh, et al., 2004). Besides determining their shopping behavior, Oh et al. (2004) has shown that tourists' social and demographic characteristics (gender and age) as well as their main motivation to travel may influence their choice of shopping outlet.

\section{Motivation to shop and shopping venues}

Besides Oh et al. (2004), numerous other studies have established that travel motivation may act as a predictor to the importance tourists assign to different types of shopping outlets (Chen, 2013; Kinley, et al., 2012; Swanson, Horridge, 2006). Chang and Chiang (2006) have segmented international tourists based on their motivation to visit Taiwan and shop at night markets. They found significant differences in the profiles of tourists belonging to different motivation segments. Similarly, focusing on senior travellers' shopping behaviour and tourism activities, Littrell, et al. (2004) found that shopping motivation was an important factor in selecting the retail venues. In conclusion, these findings suggest that market segmentation is a valid method to identify those segments within a population that are the most motivated to shop in certain types of retail venues.

\section{Shopping and other tourism activities}

Understanding the preferred combination of shopping with other tourism activities is key to developing an attractive tourist destination (Jansen-Verbeke, 1998). Most tourists, even those for which shopping is the primary motivation, engage in a wide range of activities at the destination. Knowing which activities are preferred by different types of tourist shoppers could be used by destination managers to develop appropriate marketing programmes for these segments (Pizam, et al., 1997; Swanson, Horridge, 2004).

\section{Segmentation of the Shopping Tourism Market}

Most studies using segmentation as their research method are based on two approaches: prior (apriori) segmentation (Egresi, Kara, 2015; Egresi, Polat, 2016; Hudson, 2000) and posteriori (or post-hoc or data-driven) segmentation (Dolnicar, 2004; Pesonen, et al., 2011).
Prior segmentation is based on identification of tourist market segments based on prior knowledge ('common-sense segmentation') (Dolnicar, 2004). This method is very popular in tourism studies because it is less time consuming yet still very effective for segmenting markets. Consequently, many segmentation studies are in this category, especially among the earlier ones.

Posteriori segmentation studies use factor cluster statistical analysis to identify the sizes of segments that were previously unknown (Bieger, Laesser, 2002). The outcome in this case could be much more relevant because it is based on empirically delineated segments (Bieger, Laesser, 2002).

The main criteria used for segmentation are: nationality (Jonsson, Devonish, 2008; Kozak, 2002), socio-demographics (Chen, et al., 2014; Jonsson, Devonish, 2008; Mak, et al., 1999), psychographics (Weaver, et al., 1994), tourist behaviour (Frochot, Morrison, 2000; Mak, et al., 1999; Yuksel, Yuksel, 2002), benefits (Frochot, Morrison, 2000; Pesonen, et al., 2011) and expenditure (Diaz-Perez, et al., 2005; Mok, Iverson, 2000). Segmentation of tourists by motivation is one of the most effective methods as it could be used to better understand tourists visiting certain destinations or engaging in certain forms of tourism (Park, Yoon, 2009).

Most of the time, the segmentation method was used to study motivation in mainstream (generally sea-sun-sand) tourism (for example Andreu, et al., 2005; Kozak, 2002) and only a few times was employed in studies regarding niche tourism forms, such as rural tourism (Devesa, et al., 2010; Dong, et al., 2013; Rid, et al., 2014), golf tourism (Kim, Ritchie, 2012), wellbeing tourism (Pesonen, et al., 2011), luxury shopping tourism (Park, et al., 2010), senior travel tourism (Boksberger, Laesser, 2009; Littrell, et al., 2004), nature-based tourism (Xu, Chan, 2016), responsible tourism (Mody, et al., 2014), gastronomic tourism (Lopez-Guzman, et al., 2014), and cultural event tourism (Brida, et al., 2012).

Very few studies have applied the segmentation method to study shopping tourists. For example, $\mathrm{Hu}$ and $\mathrm{Yu}$ (2007) segmented tourist shoppers based on their craft selection. They found three groups of tourist shoppers based on their selection of craft souvenirs and on their shopping involvement: shopping enthusiasts, shopping lovers, and indifferent shoppers.

Guiry et al. (2006) have also proposed a segmentation of shopping tourists. Based on the tourists' level of involvement in shopping, the authors distinguished three categories: shopping enthusiasts, normal shoppers and shopping aversives.

Profiling rural shopping travelers, Carmichael and Smith (2004) found five relevant clusters based on the motivations of these travelers: "shopping vaca- 
tion", "short vacation", get-away and visiting friends", "outdoors and sports", and "long-vacation travelers". They concluded that age, sex and the level of activity in the area were meaningful variables the segment rural shopping travelers. The study also found that those travelers who visited relatives were more likely to spend on shopping.

Bergadaa et al.'s (1995) study on shopping involvement distinguished four groups: one group that was motivated to shop for economic benefits, another group which, besides value for money, was driven to shop by the perceived social and relaxation benefits and two non-shopper groups.

Examining air passengers' shopping motivation in an international airport in Taiwan, Chung et al. (2013) found that motivations are similar to in-store shoppers elsewhere. They generated four clusters: apathetic shoppers, traditional shoppers, mood shoppers and shopping lovers.

Although motivation is one of the most popular topics in tourism studies and the segmentation method is very effective, so far there has been limited research on shopping tourism segments. This study intends to fill in this gap.

\section{Methodology}

A questionnaire was conducted between October and December 2013 with the help of five research assistants in some of the most popular tourist spots in Istanbul and on the premises of Atatürk International Airport as well as in a number of hotels. The survey targeted international tourists and the assistants were instructed to approach each $\mathrm{n}^{\text {th }}$ person, where $\mathrm{n}$ was based on the volume of human traffic in the area. If the person approached confirmed being a tourist, he or she was asked to volunteer a few minutes of his/her time to answer a few questions. If the person refused or turned out to be a local, the assistant moved on to the next person. Most tourists approached accepted to be interviewed. For those who refused, the main reason was lack of, or insufficient, knowledge of English or Turkish.

The questionnaire included 21 questions divided into four parts: 1) Socio-demographic profile; 2) Questions about the trip to and the stay in Istanbul; 3) Questions about trip motivation and activities done or planned in Istanbul; and 4) Questions about shopping attitudes and preferences.

A multiple-choice nominal scale was designed to collect information in the first part of the survey. In the second part of the questionnaire, rates of interest with shopping perceived by visitors were ascertained by using a Likert 5-point scale, ranging from 5 (strongly agree) to 1 (strongly disagree).
In the end, we collected a number of 417 questionnaires. Of these, some had missing values and were discarded and 322 were deemed usable. Almost 63\% of our respondents were from Europe (table 3), mainly from Germany ( 35 respondents), United Kingdom (31 respondents) and the Netherlands (30 respondents). Much fewer tourists originated from the Middle East and North Africa (14.6\%), other Asian countries (7.1\%), Sub-Saharan Africa (5.9\%) and the rest of the world (5.9\%). The study sample included a few more men (52.9\%) than women $(47.1 \%)$ and was dominated by younger tourists, with $63.3 \%$ of the respondents aged 20-39 (table 4). Also, most respondents were highlyeducated $(57.6 \%$ had a university diploma and $21.8 \%$ a post-graduate degree) and enjoyed good (46.9\%) and very good $(23.6 \%)$ incomes.

The data collected was subsequently processed, evaluated and explained using the latest version of SPSS software. The following statistical methods were used:

1. Factor analysis was performed to reduce the number of variables to a smaller set of factor attributes.

2. Cluster analysis was then employed to determine the number of homogenous groups formed by the data.

3. Cross tabulations using chi-square were performed to profile the clusters according to their geographical origin, demographic characteristics and travel behaviour, motivation for the trip, activity participation and shopping preferences and attitudes.

\section{Results and Discussion}

The Cronbach alpha test was first used to check the internal consistency of each factor variable of the measurement instrument. The result $(\alpha=0.788)$ showed a good level of consistency. Next we performed a Principal Component Analysis (PCA). Component factors with an eigenvalue of one or greater were rotated using the Varimax procedure. The correlation matrix in general displayed strong correlation between variables (above 0.3). The values for the Kaiser-MeyerOlkin (KMO) test (0.755) and for the Barlett's test of sphericity (0.00o) showed that the data was appropriate for the analysis. Also, eight of the 11 communalities were above 0.60 indicating a strong correlation between the variables and the associated factors. Four components had an eigenvalue of 1.0 or greater and explained $65.954 \%$ of the variance. The results are presented in table 1.

Next, in order to identify segments of tourists, cluster analysis was employed. A multi-step cluster analysis procedure was followed (Andreu, et al., 2005). The number of clusters was first determined using a hierarchical clustering method. Based on the 
Table 1. Principal Component Analysis

\begin{tabular}{|c|c|c|c|c|c|c|}
\hline Components and Items & Eigenvalue & $\begin{array}{l}\% \text { of variance } \\
\text { explained }\end{array}$ & $\begin{array}{l}\text { Item } \\
\text { loadings }\end{array}$ & Mean* & SD & $\begin{array}{l}\text { Cronbach's } \\
\text { alpha }\end{array}$ \\
\hline $\begin{array}{l}\text { Component } 1 \text { : } \\
\text { Shopping for product diversity and quality }\end{array}$ & 3.678 & 33.43 & & & & 0.636 \\
\hline 1. There are many brand stores & & & 0.735 & 3.35 & 1.124 & \\
\hline $\begin{array}{l}\text { 2. There are many shops/great variety of } \\
\text { products }\end{array}$ & & & 0.729 & 3.76 & 0.966 & \\
\hline 3. Products are of very good quality & & & 0.727 & 3.22 & 1.022 & \\
\hline $\begin{array}{l}\text { 4. There are many products I cannot find in } \\
\text { my town/country }\end{array}$ & & & 0.621 & 3.49 & 1.234 & \\
\hline $\begin{array}{l}\text { Component } 2 \text { : } \\
\text { Shopping as cultural experience }\end{array}$ & 1.414 & 12.85 & & & & 0.681 \\
\hline 1. I enjoy the cultural experience & & & 0.788 & 3.66 & 1.121 & \\
\hline 2. I am interested in local products & & & 0.783 & 3.84 & 1.095 & \\
\hline 3. The service is very good & & & 0.681 & 3.53 & 1.033 & \\
\hline $\begin{array}{l}\text { Component 3: } \\
\text { Shopping for gifts and souvenirs }\end{array}$ & 1.139 & 10.35 & & & & 0.761 \\
\hline 1. To buy gifts for family and friends & & & .894 & 3.82 & 1.201 & \\
\hline $\begin{array}{l}\text { 2. To buy souvenirs to remind me of the } \\
\text { trip }\end{array}$ & & & 0.799 & 3.72 & 1.206 & \\
\hline $\begin{array}{l}\text { Component } 4 \text { : } \\
\text { Shopping for price and value }\end{array}$ & 1.025 & 9.32 & & & & 0.632 \\
\hline $\begin{array}{l}\text { 1. Products are cheaper than in my town/ } \\
\text { country }\end{array}$ & & & 0.912 & 3.12 & 1.254 & \\
\hline 2. Good value for the money & & & 0.715 & 3.30 & 1.028 & \\
\hline
\end{tabular}

*The mean was calculated on a five-point scale where 1 meant 'strongly disagree' and 5 'strongly agree'

Ward' algorithm schedule and using the squared Euclidian distance as the measure of proximity it was determined that the five-cluster solution would be appropriate for our data (the greatest increase was observed between the five and four cluster models). Next, we used the k-means procedure (non-hi- erarchical clustering), an algorithm using the nearest centroid sorting method of clustering to actually form the clusters using the five-cluster model determined earlier (Table 2).

\section{The clusters}

Table 2. Results of the non-hierarchical clustering with the five clusters

\begin{tabular}{|c|c|c|c|c|c|c|c|}
\hline \multirow[b]{2}{*}{ Motivation component } & \multicolumn{5}{|c|}{ Cluster means } & \multirow[b]{2}{*}{ F-value } & \multirow[b]{2}{*}{ Sig. } \\
\hline & $\begin{array}{l}\text { Cluster } 1 \\
\text { (product- } \\
\text { focused } \\
\text { shoppers) }\end{array}$ & $\begin{array}{c}\text { Cluster } 2 \\
\text { (shoppers } \\
\text { for cultural } \\
\text { experience) }\end{array}$ & $\begin{array}{l}\text { Cluster } 3 \\
\text { (reluctant } \\
\text { shoppers) }\end{array}$ & $\begin{array}{c}\text { Cluster } 4 \\
\text { (difference } \\
\text { seekers) }\end{array}$ & $\begin{array}{l}\text { Cluster } \\
5 \text { (total } \\
\text { shoppers) }\end{array}$ & & \\
\hline $\begin{array}{l}\text { Shopping for product } \\
\text { diversity and quality }\end{array}$ & $\begin{array}{c}1.754 \\
\text { Very high }\end{array}$ & $\begin{array}{l}-0.260 \\
\text { Relatively low }\end{array}$ & $\begin{array}{l}-1.156 \\
\text { Very low }\end{array}$ & $\begin{array}{l}0.540 \\
\text { High }\end{array}$ & $\begin{array}{l}0.349 \\
\text { Relatively high }\end{array}$ & 62.477 & 0.000 \\
\hline $\begin{array}{l}\text { Shopping as cultural } \\
\text { experience }\end{array}$ & $\begin{array}{l}-1.833 \\
\text { Very low }\end{array}$ & $\begin{array}{l}0.183 \\
\text { Relatively high }\end{array}$ & $\begin{array}{c}-1.039 \\
\text { Very low }\end{array}$ & $\begin{array}{l}0.451 \\
\text { High }\end{array}$ & $\begin{array}{l}0.333 \\
\text { Relatively high }\end{array}$ & 64.498 & 0.000 \\
\hline $\begin{array}{l}\text { Shopping for gifts and } \\
\text { souvenirs }\end{array}$ & $\begin{array}{c}-0.235 \\
\text { Relatively low }\end{array}$ & $\begin{array}{l}-1.254 \\
\text { Very low }\end{array}$ & $\begin{array}{c}-0.257 \\
\text { Relatively low }\end{array}$ & $\begin{array}{c}-0.338 \\
\text { Relatively low }\end{array}$ & $\begin{array}{l}0.554 \\
\text { High }\end{array}$ & 62.462 & 0.000 \\
\hline $\begin{array}{l}\text { Shopping for price and } \\
\text { value }\end{array}$ & $\begin{array}{c}-0.045 \\
\text { Relatively low }\end{array}$ & $\begin{array}{c}-0.783 \\
\text { Low }\end{array}$ & $\begin{array}{l}-0.435 \\
\text { Low }\end{array}$ & $\begin{array}{l}-1.270 \\
\text { Very low }\end{array}$ & $\begin{array}{l}\quad 0.340 \\
\text { Relatively high }\end{array}$ & 69.359 & 0.000 \\
\hline Cluster size (n) & 19 & 55 & 47 & 56 & 145 & & \\
\hline $\begin{array}{l}\text { Percentage of } \\
\text { respondents (\%) }\end{array}$ & 5.90 & 17.08 & 14.60 & 17.39 & 45.03 & & \\
\hline
\end{tabular}


Table 2 describes the five clusters based on the importance of each motivation factor. The first group, which we named 'product-focused shoppers' is the smallest in size, with only 19 tourists (or $5.9 \%$ of the total number of surveyed visitors) belonging to this cluster. These tourists are highly motivated to shop for quality products that cannot be found in their home country. They care less about the price or the value of the products and are the least likely to perceive shopping as a cultural experience. They are also less interested in buying gifts and souvenirs. The second cluster includes tourists who shop at the destination mainly for the cultural experience. Perhaps for this reason, tourists in this cluster do not consider price when making their purchases. These tourists are, also, less interested in buying souvenirs (to preserve their memories) or gifts to family members and friends. In addition, for these tourists, diversity and quality of products is less important when shopping. The third group (labelled 'reluctant shoppers') is made up of those tourists who are not motivated to shop ( 47 individuals or $14.6 \%$ of the total) by any of the four factors and shop only when they have to. Tourists included in the fourth segment ('difference seekers') are highly motivated to shop by their interest in good quality products that cannot be found in their places of origin. When making their choice they do not consider price or overall value of the product and have relatively little interest in buying souvenirs or gifts. However, unlike tourists in cluster 1 , these tourists also see shopping as a cultural experience when visiting destinations away from home. Finally, almost half of all tourists surveyed (145 or $45 \%$ ) turned out to be highly or relatively highly interested in shopping for all four motivation categories. For this reason, we labelled them 'total shoppers'. These tourists were particularly interested in buying souvenirs for themselves and gifts for their relatives and friends back home.

Table 3. Geographical origin of tourists by cluster

\section{Differences between clusters}

Next, using results from chi-square tests, the following tables report on the differences in geographical origin (table 3), in socio-demographic characteristics (table 4), in travel characteristics (table 5), in motivation to visit (table 6), in the importance tourists assign to different forms of shopping outlets (table 7 ) and in the tourists' perception of shopping benefits (table 8) between the five clusters obtained.

The results of our research show that, similar to other studies (Brida, et al., 2012; D'Urso, et al., 2016; D'Urso, et al., 2013; Littrell, et al., 2004, Mody, et al., 2014; Tkaczynski, et al., 2009), tourists' country of origin is an important indicator for tourists' shopping typologies. Table 3 shows that while Europeans are the largest group in all five clusters (they represent $62.7 \%$ of all tourists in our survey), they form the majority only among 'shoppers for cultural experience', 'reluctant shoppers' and 'total shoppers'. Tourists from the Middle East and North Africa constituted a significant minority among 'product-focused shoppers' and 'difference seekers'. We should also note that tourists from Sub-Saharan Africa (and, to a lesser degree, tourists from the Middle East and North Africa) are over-represented in the first cluster ('product-focused shoppers') while a much higher percentage of Europeans than the average belong to clusters 2 ('shoppers for cultural experience') and 5 ('total shoppers').

While other researchers argue that socio-demographic characteristics such as gender (Andreu, et al., 2005; Brida, et al., 2012; Hu, Yu, 2007; Rid, et al., 2014), age (Brida, et al., 2012; Carmichael, Smith, 2004; Lopez-Guzman, et al., 2014; Mody, et al., 2014; Rid, et al., 2014), education (Carmichael, Smith, 2004; D'Urso et al., 2013; Lopez-Guzman et al., 2014; Park, Yoon, 2009) or income (Andreu, et al., 2005; Brida, et al., 2012; Carmichael, Smith, 2004; D'Urso, et al., 2013; Lopez-Guzman, et al., 2014) could generate statistical-

\begin{tabular}{|c|c|c|c|c|c|c|c|c|c|}
\hline Variable & Description & $C 1^{*}$ & $\mathrm{C} 2$ & C 3 & C 4 & C 5 & $\begin{array}{l}\text { Total } \\
\text { column }\end{array}$ & $\begin{array}{l}\text { Pearson's Chi- } \\
\text { square Value }\end{array}$ & Df \\
\hline \multirow{6}{*}{$\begin{array}{l}\text { Region** } \\
p=0.001\end{array}$} & Europe & $\begin{array}{l}36.8 \\
(3.5)\end{array}$ & $\begin{array}{c}74.4 \\
(20.3)\end{array}$ & $\begin{array}{c}57.2 \\
(13.4)\end{array}$ & $\begin{array}{c}41.1 \\
(11.4)\end{array}$ & $\begin{array}{l}71.7 \\
(51.5)\end{array}$ & $\begin{array}{c}62.7 \\
(100.0)\end{array}$ & \multirow{6}{*}{45.119} & \multirow{6}{*}{20} \\
\hline & $\begin{array}{l}\text { Middle East } \\
\text { and North Africa }\end{array}$ & $\begin{array}{c}31.6 \\
(12.8)\end{array}$ & $\begin{array}{c}3.6 \\
(4.3)\end{array}$ & $\begin{array}{c}17.0 \\
(17.0)\end{array}$ & $\begin{array}{l}26.8 \\
(31.9)\end{array}$ & $\begin{array}{c}11.0 \\
(34.0)\end{array}$ & $\begin{array}{c}14.6 \\
(100.0)\end{array}$ & & \\
\hline & $\begin{array}{l}\text { Other Asian } \\
\text { countries }\end{array}$ & $\begin{array}{l}10.5 \\
(8.7)\end{array}$ & $\begin{array}{c}9.1 \\
(21.7)\end{array}$ & $\begin{array}{c}6.4 \\
(13.0)\end{array}$ & $\begin{array}{l}10.7 \\
(26.1)\end{array}$ & $\begin{array}{c}4.8 \\
(30.4)\end{array}$ & $\begin{array}{c}7.1 \\
(100.0)\end{array}$ & & \\
\hline & Sub-Saharan Africa & $\begin{array}{c}21.1 \\
(21.1)\end{array}$ & $\begin{array}{c}3.6 \\
(10.5)\end{array}$ & $\begin{array}{c}8.5 \\
(21.1)\end{array}$ & $\begin{array}{c}10.7 \\
(31.6)\end{array}$ & $\begin{array}{c}2.1 \\
(15.8)\end{array}$ & $\begin{array}{c}5.9 \\
(100.0)\end{array}$ & & \\
\hline & Rest of the world & $\begin{array}{c}0.0 \\
(0.0)\end{array}$ & $\begin{array}{c}3.6 \\
(10.5)\end{array}$ & $\begin{array}{c}6.4 \\
(15.8)\end{array}$ & $\begin{array}{c}7.1 \\
(21.1)\end{array}$ & $\begin{array}{c}6.9 \\
(52.6)\end{array}$ & $\begin{array}{c}5.9 \\
(100.0)\end{array}$ & & \\
\hline & Not specified & $\begin{array}{c}0.0 \\
(0.0)\end{array}$ & $\begin{array}{c}5.5 \\
(25.0)\end{array}$ & $\begin{array}{c}4.3 \\
(16.7)\end{array}$ & $\begin{array}{c}3.6 \\
(16.7)\end{array}$ & $\begin{array}{c}3.4 \\
(41.7)\end{array}$ & $\begin{array}{c}3.7 \\
(100.0)\end{array}$ & & \\
\hline
\end{tabular}

* Percent within variable (percent within cluster number of case); ** Statistically significant at $p<0.01$ 
Tourist Market Segmentation by Motivation to Shop:

A Case Study of Istanbul, Turkey

Table 4. Socio-demographic characteristics of tourists by cluster

\begin{tabular}{|c|c|c|c|c|c|c|c|c|c|}
\hline Variable & Description & $\mathrm{C} 1 *$ & $\mathrm{C} 2$ & $\mathrm{C} 3$ & $\mathrm{C} 4$ & $\mathrm{C} 5$ & $\begin{array}{l}\text { Total } \\
\text { column }\end{array}$ & $\begin{array}{l}\text { Person's Chi- } \\
\text { square value }\end{array}$ & Df \\
\hline \multirow{2}{*}{$\begin{array}{l}\text { Gender } \\
p=0.234 \\
>.05\end{array}$} & Male & $\begin{array}{l}72.2 \\
(7.9)\end{array}$ & $\begin{array}{l}58.5 \\
(18.9)\end{array}$ & $\begin{array}{l}52.2 \\
(14.6)\end{array}$ & $\begin{array}{l}44.6 \\
(15.2)\end{array}$ & $\begin{array}{l}49.0 \\
(43.3)\end{array}$ & $\begin{array}{c}52.9 \\
(100.0)\end{array}$ & \multirow{2}{*}{5.567} & \multirow{2}{*}{4} \\
\hline & Female & $\begin{array}{l}27.8 \\
(3.2) \\
\end{array}$ & $\begin{array}{c}41.5 \\
(14.8)\end{array}$ & $\begin{array}{c}47.8 \\
(14.2) \\
\end{array}$ & $\begin{array}{c}55.4 \\
(20.0) \\
\end{array}$ & $\begin{array}{c}51.0 \\
(47.7) \\
\end{array}$ & $\begin{array}{c}47.1 \\
(100.0)\end{array}$ & & \\
\hline \multirow{4}{*}{$\begin{array}{l}\text { Age } \\
p=0.623 \\
>0.05\end{array}$} & $<20$ & $\begin{array}{l}10.5 \\
(5.0) \\
\end{array}$ & $\begin{array}{c}10.9 \\
(15.0)\end{array}$ & $\begin{array}{c}17.0 \\
(20.0)\end{array}$ & $\begin{array}{c}17.9 \\
(25.0)\end{array}$ & $\begin{array}{c}9.7 \\
(35.0)\end{array}$ & $\begin{array}{c}10.6 \\
(100.0)\end{array}$ & \multirow{4}{*}{9.801} & \multirow{4}{*}{12} \\
\hline & $20-39$ & $\begin{array}{l}63.2 \\
(5.5)\end{array}$ & $\begin{array}{c}67.3 \\
(17.0)\end{array}$ & $\begin{array}{c}57.5 \\
(12.4)\end{array}$ & $\begin{array}{c}67.8 \\
(17.6) \\
\end{array}$ & $\begin{array}{c}71.0 \\
(47.5)\end{array}$ & $\begin{array}{c}63.3 \\
(100.0)\end{array}$ & & \\
\hline & $40-59$ & $\begin{array}{l}21.0 \\
(6.7)\end{array}$ & $\begin{array}{c}20.0 \\
(18.3)\end{array}$ & $\begin{array}{c}25.5 \\
(20.0)\end{array}$ & $\begin{array}{c}14.3 \\
(13.3)\end{array}$ & $\begin{array}{c}17.2 \\
(41.7)\end{array}$ & $\begin{array}{c}20.9 \\
(100.0)\end{array}$ & & \\
\hline & $60+$ & $\begin{array}{c}5.3 \\
(20.0)\end{array}$ & $\begin{array}{c}1.8 \\
(20.0)\end{array}$ & $\begin{array}{c}0.0 \\
(0.0)\end{array}$ & $\begin{array}{c}0.0 \\
(0.0)\end{array}$ & $\begin{array}{c}2.1 \\
(60.0)\end{array}$ & $\begin{array}{c}5.3 \\
(100.0)\end{array}$ & & \\
\hline \multirow{4}{*}{$\begin{array}{l}\text { Education } \\
p=0.305 \\
>0.05\end{array}$} & Less than high school & $\begin{array}{c}5.3 \\
(5.9)\end{array}$ & $\begin{array}{c}9.1 \\
(29.4)\end{array}$ & $\begin{array}{c}4.3 \\
(11.8)\end{array}$ & $\begin{array}{c}10.7 \\
(35.5)\end{array}$ & $\begin{array}{c}2.1 \\
(17.6)\end{array}$ & $\begin{array}{c}5.0 \\
(100.0)\end{array}$ & \multirow{4}{*}{13.926} & \multirow{4}{*}{12} \\
\hline & High school & $\begin{array}{l}10.5 \\
(4.0)\end{array}$ & $\begin{array}{c}18.2 \\
(20.0)\end{array}$ & $\begin{array}{c}21.3 \\
(20.0)\end{array}$ & $\begin{array}{c}7.1 \\
(8.0)\end{array}$ & $\begin{array}{c}16.6 \\
(48.0)\end{array}$ & $\begin{array}{c}15.6 \\
(100.0)\end{array}$ & & \\
\hline & University & $\begin{array}{l}57.9 \\
(6.0) \\
\end{array}$ & $\begin{array}{c}52.7 \\
(15.9) \\
\end{array}$ & $\begin{array}{l}46.8 \\
(12.1) \\
\end{array}$ & $\begin{array}{c}60.7 \\
(18.7) \\
\end{array}$ & $\begin{array}{c}59.3 \\
(47.3) \\
\end{array}$ & $\begin{array}{c}57.6 \\
(100.0)\end{array}$ & & \\
\hline & Post graduate & $\begin{array}{l}36.3 \\
(6.8)\end{array}$ & $\begin{array}{l}20.0 \\
(15.1)\end{array}$ & $\begin{array}{c}27.6 \\
(17.8)\end{array}$ & $\begin{array}{c}21.5 \\
(16.4)\end{array}$ & $\begin{array}{c}22.0 \\
(43.8)\end{array}$ & $\begin{array}{c}21.8 \\
(100.0)\end{array}$ & & \\
\hline \multirow{4}{*}{$\begin{array}{l}\text { Income } \\
p=0.111 \\
>0.05\end{array}$} & Very good & $\begin{array}{l}22.2 \\
(5.0)\end{array}$ & $\begin{array}{c}15.1 \\
(10.0)\end{array}$ & $\begin{array}{c}18.2 \\
(10.0)\end{array}$ & $\begin{array}{c}19.6 \\
(13.8)\end{array}$ & $\begin{array}{c}35.0 \\
(61.2)\end{array}$ & $\begin{array}{c}23.6 \\
(100.0)\end{array}$ & \multirow{2}{*}{18.151} & \multirow{2}{*}{12} \\
\hline & Good & $\begin{array}{l}55.5 \\
(7.0)\end{array}$ & $\begin{array}{c}56.6 \\
(21.0)\end{array}$ & $\begin{array}{c}47.7 \\
(14.7)\end{array}$ & $\begin{array}{c}48.2 \\
(18.9)\end{array}$ & $\begin{array}{c}39.3 \\
(38.5)\end{array}$ & $\begin{array}{c}46.9 \\
(100.0)\end{array}$ & & \\
\hline & Satisfactory & $\begin{array}{c}11.1 \\
(3.0)\end{array}$ & $\begin{array}{c}17.0 \\
(13.6) \\
\end{array}$ & $\begin{array}{c}29.5 \\
(19.7) \\
\end{array}$ & $\begin{array}{c}23.2 \\
(19.7) \\
\end{array}$ & $\begin{array}{c}20.7 \\
(20.3) \\
\end{array}$ & $\begin{array}{c}21.1 \\
(100.0)\end{array}$ & & \\
\hline & Not satisfactory & $\begin{array}{l}11.1 \\
(9.1)\end{array}$ & $\begin{array}{c}11.3 \\
(27.3)\end{array}$ & $\begin{array}{l}4.6 \\
(9.1)\end{array}$ & $\begin{array}{c}9.0 \\
(22.7)\end{array}$ & $\begin{array}{c}5.0 \\
(31.8)\end{array}$ & $\begin{array}{c}8.4 \\
(100.0)\end{array}$ & & \\
\hline
\end{tabular}

* Percent within variable (percent within cluster number of case)

Table 5. Tourist travel characteristics by cluster

\begin{tabular}{|c|c|c|c|c|c|c|c|c|c|}
\hline Variable & Description & $\mathrm{C} 1 *$ & $\mathrm{C} 2$ & C3 & $\mathrm{C} 4$ & $\mathrm{C} 5$ & $\begin{array}{c}\text { Total } \\
\text { column }\end{array}$ & $\begin{array}{l}\text { Person's Chi- } \\
\text { square value }\end{array}$ & Df \\
\hline \multirow{4}{*}{$\begin{array}{l}\text { Number } \\
\text { of persons } \\
\text { travelling** } \\
p=0.000\end{array}$} & Alone & $\begin{array}{c}63.2 \\
(17.4)\end{array}$ & $\begin{array}{c}18.2 \\
(14.5)\end{array}$ & $\begin{array}{l}21.3 \\
(14.5)\end{array}$ & $\begin{array}{c}33.9 \\
(27.5)\end{array}$ & $\begin{array}{l}12.5 \\
(26.1)\end{array}$ & $\begin{array}{c}20.9 \\
(100.0)\end{array}$ & \multirow{4}{*}{41.283} & \multirow{4}{*}{12} \\
\hline & Friends (up to 4) & $\begin{array}{l}21.1 \\
(3.5)\end{array}$ & $\begin{array}{c}34.5 \\
(16.0)\end{array}$ & $\begin{array}{c}44.7 \\
(18.3)\end{array}$ & $\begin{array}{l}26.8 \\
(13.0)\end{array}$ & $\begin{array}{c}38.9 \\
(48.7)\end{array}$ & $\begin{array}{c}33.4 \\
(100.0)\end{array}$ & & \\
\hline & Family (up to 4) & $\begin{array}{l}10.5 \\
(1.9) \\
\end{array}$ & $\begin{array}{c}36.4 \\
(19.0)\end{array}$ & $\begin{array}{l}17.0 \\
(7.6) \\
\end{array}$ & $\begin{array}{c}33.9 \\
(18.1)\end{array}$ & $\begin{array}{c}38.9 \\
(53.3)\end{array}$ & $\begin{array}{c}34.9 \\
(100.0)\end{array}$ & & \\
\hline & Group (more than 4) & $\begin{array}{c}5.3 \\
(3.0)\end{array}$ & $\begin{array}{c}10.9 \\
(18.2)\end{array}$ & $\begin{array}{c}17.0 \\
(24.2)\end{array}$ & $\begin{array}{l}5.4 \\
(9.1) \\
\end{array}$ & $\begin{array}{c}9.7 \\
(45.5)\end{array}$ & $\begin{array}{c}10.8 \\
(100.0)\end{array}$ & & \\
\hline \multirow{4}{*}{$\begin{array}{l}\text { Means of } \\
\text { travel } \\
p=0.295 \\
>0.05\end{array}$} & Air & $\begin{array}{l}94.7 \\
(5.8)\end{array}$ & $\begin{array}{l}98.2 \\
(17.5)\end{array}$ & $\begin{array}{c}95.7 \\
(14.6)\end{array}$ & $\begin{array}{c}92.9 \\
(16.8)\end{array}$ & $\begin{array}{c}97.2 \\
(45.3)\end{array}$ & $\begin{array}{c}95.7 \\
(100.0)\end{array}$ & \multirow{4}{*}{14.090} & \multirow{4}{*}{12} \\
\hline & Train & $0.0(0.0)$ & $\begin{array}{c}1.8 \\
(33.3)\end{array}$ & $\begin{array}{c}2.1 \\
(33.3)\end{array}$ & $\begin{array}{c}1.8 \\
(33.3)\end{array}$ & $0.0(0.0)$ & $\begin{array}{c}1.7 \\
(100.0)\end{array}$ & & \\
\hline & Bus & $\begin{array}{c}5.3 \\
(12.5)\end{array}$ & $0.0(0.0)$ & $0.0(0.0)$ & $\begin{array}{c}5.4 \\
(37.5)\end{array}$ & $\begin{array}{c}2.8 \\
(50.0)\end{array}$ & $\begin{array}{c}2.2 \\
(100.0) \\
\end{array}$ & & \\
\hline & Car & $0.0(0.0)$ & $0.0(0.0)$ & $\begin{array}{c}2.1 \\
(100.0)\end{array}$ & $0.0(0.0)$ & $0.0(0.0)$ & $\begin{array}{c}0.5 \\
(100.0)\end{array}$ & & \\
\hline
\end{tabular}




\begin{tabular}{|c|c|c|c|c|c|c|c|c|c|}
\hline Variable & Description & $\mathrm{C} 1^{*}$ & $\mathrm{C} 2$ & $\mathrm{C} 3$ & $\mathrm{C} 4$ & $\mathrm{C} 5$ & $\begin{array}{l}\text { Total } \\
\text { column }\end{array}$ & $\begin{array}{l}\text { Person's Chi- } \\
\text { square value }\end{array}$ & Df \\
\hline \multirow{5}{*}{$\begin{array}{l}\text { Accommo- } \\
\text { dation } \\
p=0.056 \\
>0.05\end{array}$} & Hotel $4-5^{*}$ & $\begin{array}{l}55.6 \\
(7.9) \\
\end{array}$ & $\begin{array}{c}35.2 \\
(15.0)\end{array}$ & $\begin{array}{l}30.4 \\
(11.0)\end{array}$ & $\begin{array}{c}42.9 \\
(18.9)\end{array}$ & $\begin{array}{c}41.4 \\
(47.2)\end{array}$ & $\begin{array}{c}38.3 \\
(100.0)\end{array}$ & \multirow{5}{*}{25.862} & \multirow{5}{*}{16} \\
\hline & Hotel 1-3* & $\begin{array}{l}11.1 \\
(2.7) \\
\end{array}$ & $\begin{array}{l}20.4 \\
(14.7)\end{array}$ & $\begin{array}{c}19.6 \\
(12.0) \\
\end{array}$ & $\begin{array}{l}25.0 \\
(18.7) \\
\end{array}$ & $\begin{array}{c}26.9 \\
(52.0) \\
\end{array}$ & $\begin{array}{c}24.5 \\
(100.0)\end{array}$ & & \\
\hline & $\begin{array}{l}\text { Other } \\
\text { (hostel, motel, etc.) }\end{array}$ & $\begin{array}{c}5.6 \\
(1.6) \\
\end{array}$ & $\begin{array}{c}18.5 \\
(15.6) \\
\end{array}$ & $\begin{array}{c}32.6 \\
(23.4) \\
\end{array}$ & $\begin{array}{c}19.6 \\
(17.2)\end{array}$ & $\begin{array}{c}18.6 \\
(42.2) \\
\end{array}$ & $\begin{array}{c}20.3 \\
(100.0)\end{array}$ & & \\
\hline & $\begin{array}{l}\text { With family and } \\
\text { friends }\end{array}$ & $\begin{array}{c}5.6 \\
(3.3) \\
\end{array}$ & $\begin{array}{c}18.5 \\
(33.3)\end{array}$ & $\begin{array}{c}8.7 \\
(13.3)\end{array}$ & $\begin{array}{c}3.6 \\
(6.7) \\
\end{array}$ & $\begin{array}{c}9.0 \\
(43.3)\end{array}$ & $\begin{array}{c}9.0 \\
(100.0)\end{array}$ & & \\
\hline & No accommo-dation & $\begin{array}{l}22.2 \\
(17.4) \\
\end{array}$ & $\begin{array}{c}7.4 \\
(17.4)\end{array}$ & $\begin{array}{c}8.7 \\
(17.4)\end{array}$ & $\begin{array}{c}8.9 \\
(21.7)\end{array}$ & $\begin{array}{c}4.1 \\
(26.1)\end{array}$ & $\begin{array}{c}8.0 \\
(100.0)\end{array}$ & & \\
\hline \multirow{4}{*}{$\begin{array}{l}\text { Number of } \\
\text { previous } \\
\text { visits** } \\
p=0.000\end{array}$} & Many times & $\begin{array}{l}36.8 \\
(17.9)\end{array}$ & $\begin{array}{c}10.9 \\
(15.4)\end{array}$ & $\begin{array}{c}8.5 \\
(10.3)\end{array}$ & $\begin{array}{c}16.4 \\
(23.1)\end{array}$ & $\begin{array}{c}9.0 \\
(33.3)\end{array}$ & $\begin{array}{c}12.1 \\
(100.0) \\
\end{array}$ & \multirow{4}{*}{35.289} & \multirow{4}{*}{12} \\
\hline & A few times & $\begin{array}{l}26.3 \\
(17.9)\end{array}$ & $\begin{array}{l}3.6 \\
(7.1)\end{array}$ & $\begin{array}{c}10.6 \\
(17.9)\end{array}$ & $\begin{array}{c}10.9 \\
(21.4)\end{array}$ & $\begin{array}{c}6.9 \\
(35.7)\end{array}$ & $\begin{array}{c}8.7 \\
(100.0)\end{array}$ & & \\
\hline & One time & $\begin{array}{l}10.5 \\
(6.3) \\
\end{array}$ & $\begin{array}{c}5.5 \\
(9.4) \\
\end{array}$ & $\begin{array}{c}17.0 \\
(25.0)\end{array}$ & $\begin{array}{c}14.5 \\
(25.0)\end{array}$ & $\begin{array}{c}7.6 \\
(34.4)\end{array}$ & $\begin{array}{c}10.0 \\
(100.0)\end{array}$ & & \\
\hline & Never & $\begin{array}{l}26.3 \\
(2.3)\end{array}$ & $\begin{array}{c}80.0 \\
(19.8)\end{array}$ & $\begin{array}{c}63.8 \\
(13.5)\end{array}$ & $\begin{array}{c}58.2 \\
(14.4)\end{array}$ & $\begin{array}{c}76.5 \\
(50.0)\end{array}$ & $\begin{array}{c}62.2 \\
(100.0)\end{array}$ & & \\
\hline \multirow{4}{*}{$\begin{array}{l}\text { Length of } \\
\text { stay*** } \\
p=0.034\end{array}$} & More than a week & $\begin{array}{l}10.5 \\
(4.3)\end{array}$ & $\begin{array}{c}16.4 \\
(19.1)\end{array}$ & $\begin{array}{c}8.5 \\
(8.5)\end{array}$ & $\begin{array}{c}19.6 \\
(23.4)\end{array}$ & $\begin{array}{c}14.5 \\
(44.7)\end{array}$ & $\begin{array}{c}14.6 \\
(100.0)\end{array}$ & \multirow{4}{*}{22.351} & \multirow{4}{*}{12} \\
\hline & 3-7 nights & $\begin{array}{l}47.4 \\
(4.8)\end{array}$ & $\begin{array}{c}58.2 \\
(17.0)\end{array}$ & $\begin{array}{l}46.8 \\
(11.7)\end{array}$ & $\begin{array}{c}51.8 \\
(15.4)\end{array}$ & $\begin{array}{l}66.2 \\
(51.1)\end{array}$ & $\begin{array}{c}58.4 \\
(100.0)\end{array}$ & & \\
\hline & $1-2$ nights & $\begin{array}{l}21.0 \\
(6.6) \\
\end{array}$ & $\begin{array}{c}21.8 \\
(19.7) \\
\end{array}$ & $\begin{array}{c}34.0 \\
(26.2) \\
\end{array}$ & $\begin{array}{c}10.1 \\
(34.6)\end{array}$ & $\begin{array}{c}13.8 \\
(32.8) \\
\end{array}$ & $\begin{array}{c}18.9 \\
(100.0)\end{array}$ & & \\
\hline & A few hours & $\begin{array}{c}21.0 \\
(15.4)\end{array}$ & $\begin{array}{c}3.6 \\
(7.7)\end{array}$ & $\begin{array}{c}10.6 \\
(19.2)\end{array}$ & $\begin{array}{c}12.5 \\
(26.9)\end{array}$ & $\begin{array}{c}5.5 \\
(30.7)\end{array}$ & $\begin{array}{c}8.1 \\
(100.0)\end{array}$ & & \\
\hline
\end{tabular}

*Percent within variable (percent within cluster number of case); ** Statistically significant at $p<0.01$; ** Statistically significant at $p<0.05$

Table 6. Motivation to visit Istanbul and activities done or planned to be done while in Istanbul, by cluster

\begin{tabular}{|c|c|c|c|c|c|c|c|c|c|}
\hline Variable & Description & $C 1^{*}$ & $C 2$ & C 3 & C 4 & $C 5$ & $\begin{array}{l}\text { Total } \\
\text { column }\end{array}$ & $\begin{array}{l}\text { Pearson's Chi- } \\
\text { square Value }\end{array}$ & Df \\
\hline \multirow{4}{*}{$\begin{array}{l}\text { Primary } \\
\text { motivation } \\
\text { for visit** } \\
p=0.003\end{array}$} & Business & $\begin{array}{l}36.8 \\
(13.2)\end{array}$ & $\begin{array}{c}20.0 \\
(20.7)\end{array}$ & $\begin{array}{l}12.8 \\
(11.3)\end{array}$ & $\begin{array}{c}12.5 \\
(13.2)\end{array}$ & $\begin{array}{c}15.2 \\
(41.5)\end{array}$ & $\begin{array}{c}19.9 \\
(100.0)\end{array}$ & \multirow{4}{*}{29.582} & \multirow{4}{*}{12} \\
\hline & Pleasure & $\begin{array}{l}36.8 \\
(3.1)\end{array}$ & $\begin{array}{l}60.0 \\
(14.8)\end{array}$ & $\begin{array}{c}68.1 \\
(14.3)\end{array}$ & $\begin{array}{l}71.4 \\
(17.9)\end{array}$ & $\begin{array}{c}76.5 \\
(49.8)\end{array}$ & $\begin{array}{c}65.9 \\
(100.0)\end{array}$ & & \\
\hline & $\begin{array}{l}\text { Visiting family and } \\
\text { friends }\end{array}$ & $\begin{array}{c}0.0 \\
(0.0)\end{array}$ & $\begin{array}{c}12.7 \\
(30.4)\end{array}$ & $\begin{array}{c}8.5 \\
(17.4)\end{array}$ & $\begin{array}{c}8.9 \\
(21.7)\end{array}$ & $\begin{array}{c}4.8 \\
(30.4)\end{array}$ & $\begin{array}{c}7.0 \\
(100.0)\end{array}$ & & \\
\hline & Transit & $\begin{array}{l}26.3 \\
(21.7)\end{array}$ & $\begin{array}{c}7.3 \\
(17.4)\end{array}$ & $\begin{array}{c}10.6 \\
(21.7)\end{array}$ & $\begin{array}{c}7.1 \\
(17.4)\end{array}$ & $\begin{array}{c}3.4 \\
(21.7)\end{array}$ & $\begin{array}{c}7.2 \\
(100.0)\end{array}$ & & \\
\hline \multirow{3}{*}{$\begin{array}{l}\text { Pleasure } \\
\text { as primary } \\
\text { motive** } \\
p=0.004\end{array}$} & $\begin{array}{l}\text { Visit historical/ } \\
\text { Cultural sites }\end{array}$ & $\begin{array}{l}14.3 \\
(0.9)\end{array}$ & $\begin{array}{c}38.9 \\
(12.6)\end{array}$ & $\begin{array}{l}55.9 \\
(17.1)\end{array}$ & $\begin{array}{l}40.5 \\
(15.3)\end{array}$ & $\begin{array}{c}53.1 \\
(54.1)\end{array}$ & $\begin{array}{c}47.8 \\
(100.0)\end{array}$ & \multirow{3}{*}{28.865} & \multirow{3}{*}{12} \\
\hline & Shopping & $\begin{array}{c}57.1 \\
(16.0) \\
\end{array}$ & $\begin{array}{c}13.9 \\
(20.0) \\
\end{array}$ & $\begin{array}{c}8.8 \\
(12.0) \\
\end{array}$ & $\begin{array}{c}9.5 \\
(16.0) \\
\end{array}$ & $\begin{array}{c}8.0 \\
(36.0)\end{array}$ & $\begin{array}{c}10.8 \\
(100.0)\end{array}$ & & \\
\hline & $\begin{array}{l}\text { Experience new } \\
\text { cultures }\end{array}$ & $\begin{array}{l}28.6 \\
(2.1)\end{array}$ & $\begin{array}{c}47.2 \\
(17.7)\end{array}$ & $\begin{array}{c}35.3 \\
(12.5)\end{array}$ & $\begin{array}{l}50.0 \\
(21.9)\end{array}$ & $\begin{array}{c}38.9 \\
(45.8)\end{array}$ & $\begin{array}{c}41.4 \\
(100.0)\end{array}$ & & \\
\hline \multirow{2}{*}{$\begin{array}{l}\text { Visit the } \\
\text { main } \\
\text { historical } \\
\text { sites** } \\
p=0.000\end{array}$} & no & $\begin{array}{c}42.1 \\
(17.0)\end{array}$ & $\begin{array}{l}5.6 \\
(6.4)\end{array}$ & $\begin{array}{c}38.2 \\
(27.7)\end{array}$ & $\begin{array}{c}19.6 \\
(23.4)\end{array}$ & $\begin{array}{c}8.3 \\
(25.5) \\
\end{array}$ & $\begin{array}{c}14.6 \\
(100.0)\end{array}$ & \multirow{2}{*}{27.229} & \multirow{2}{*}{4} \\
\hline & yes & $\begin{array}{l}57.9 \\
(4.0)\end{array}$ & $\begin{array}{c}94.4 \\
(18.6)\end{array}$ & $\begin{array}{c}61.8 \\
(12.4)\end{array}$ & $\begin{array}{c}80.4 \\
(16.4)\end{array}$ & $\begin{array}{c}91.7 \\
(48.5)\end{array}$ & $\begin{array}{c}85.4 \\
(100.0)\end{array}$ & & \\
\hline
\end{tabular}




\begin{tabular}{|c|c|c|c|c|c|c|c|c|c|}
\hline Variable & Description & $C 1^{*}$ & $C 2$ & C 3 & C 4 & $C 5$ & $\begin{array}{l}\text { Total } \\
\text { column }\end{array}$ & $\begin{array}{l}\text { Pearson's Chi- } \\
\text { square Value }\end{array}$ & Df \\
\hline \multirow{2}{*}{$\begin{array}{l}\text { Take a tour } \\
\text { of the Bos- } \\
\text { porus*** } \\
p=0.049\end{array}$} & no & $\begin{array}{l}42.1 \\
(6.2)\end{array}$ & $\begin{array}{c}44.1 \\
(18.6)\end{array}$ & $\begin{array}{l}42.6 \\
(15.5)\end{array}$ & $\begin{array}{c}55.4 \\
(24.0)\end{array}$ & $\begin{array}{c}31.7 \\
(35.7)\end{array}$ & $\begin{array}{c}40.2 \\
(100.0)\end{array}$ & \multirow{2}{*}{15.516} & \multirow{2}{*}{4} \\
\hline & yes & $\begin{array}{l}57.9 \\
(5.8)\end{array}$ & $\begin{array}{c}55.9 \\
(15.7) \\
\end{array}$ & $\begin{array}{c}57.4 \\
(14.1) \\
\end{array}$ & $\begin{array}{l}44.6 \\
(13.1) \\
\end{array}$ & $\begin{array}{c}68.3 \\
(51.8) \\
\end{array}$ & $\begin{array}{c}59.8 \\
(100.0)\end{array}$ & & \\
\hline \multirow{2}{*}{$\begin{array}{l}\text { Participate in } \\
\text { sport events } \\
p=0.391\end{array}$} & no & $\begin{array}{l}84.2 \\
(5.3)\end{array}$ & $\begin{array}{c}92.6 \\
(16.8)\end{array}$ & $\begin{array}{c}95.7 \\
(14.9)\end{array}$ & $\begin{array}{l}94.6 \\
(17.5)\end{array}$ & $\begin{array}{c}95.2 \\
(45.5)\end{array}$ & $\begin{array}{c}94.1 \\
(100.0)\end{array}$ & \multirow{2}{*}{4.114} & \multirow{2}{*}{4} \\
\hline & yes & $\begin{array}{c}15.8 \\
(15.8) \\
\end{array}$ & $\begin{array}{c}7.4 \\
(21.0) \\
\end{array}$ & $\begin{array}{c}4.3 \\
(10.5) \\
\end{array}$ & $\begin{array}{c}5.4 \\
(15.8) \\
\end{array}$ & $\begin{array}{c}4.8 \\
(36.8) \\
\end{array}$ & $\begin{array}{c}5.9 \\
(100.0)\end{array}$ & & \\
\hline \multirow{2}{*}{$\begin{array}{l}\text { Participate } \\
\text { in cultural } \\
\text { events*** } \\
p=0.032\end{array}$} & no & $\begin{array}{l}78.9 \\
(6.0) \\
\end{array}$ & $\begin{array}{c}63.0 \\
(13.7) \\
\end{array}$ & $\begin{array}{c}91.5 \\
(17.3) \\
\end{array}$ & $\begin{array}{c}94.6 \\
(17.3) \\
\end{array}$ & $\begin{array}{c}78.6 \\
(45.8) \\
\end{array}$ & $\begin{array}{c}77.6 \\
(100.0) \\
\end{array}$ & \multirow{2}{*}{16.847} & \multirow{2}{*}{4} \\
\hline & yes & $\begin{array}{l}21.1 \\
(5.6)\end{array}$ & $\begin{array}{c}37.0 \\
(28.2)\end{array}$ & $\begin{array}{l}18.5 \\
(5.6)\end{array}$ & $\begin{array}{c}5.4 \\
(18.3)\end{array}$ & $\begin{array}{c}21.4 \\
(43.7)\end{array}$ & $\begin{array}{c}22.4 \\
(100.0)\end{array}$ & & \\
\hline \multirow{2}{*}{$\begin{array}{l}\text { Participate in } \\
\text { a conference } \\
p=0.353\end{array}$} & no & $\begin{array}{l}78.9 \\
(5.1) \\
\end{array}$ & $\begin{array}{c}92.6 \\
(17.0) \\
\end{array}$ & $\begin{array}{c}93.6 \\
(15.0) \\
\end{array}$ & $\begin{array}{c}91.1 \\
(17.3)\end{array}$ & $\begin{array}{c}92.4 \\
(45.6)\end{array}$ & $\begin{array}{c}91.6 \\
(100.0)\end{array}$ & \multirow{2}{*}{4.411} & \multirow{2}{*}{4} \\
\hline & yes & $\begin{array}{c}21.1 \\
(14.8) \\
\end{array}$ & $\begin{array}{c}7.4 \\
(14.8) \\
\end{array}$ & $\begin{array}{c}6.4 \\
(11.1)\end{array}$ & $\begin{array}{c}8.9 \\
(18.5) \\
\end{array}$ & $\begin{array}{c}7.6 \\
(40.7) \\
\end{array}$ & $\begin{array}{c}8.4 \\
(100.0)\end{array}$ & & \\
\hline \multirow{2}{*}{$\begin{array}{l}\text { Visit an } \\
\text { exhibition } \\
p=0.878\end{array}$} & no & $\begin{array}{l}78.9 \\
(5.8) \\
\end{array}$ & $\begin{array}{c}77.8 \\
(16.3) \\
\end{array}$ & $\begin{array}{c}76.6 \\
(14.0) \\
\end{array}$ & $\begin{array}{l}80.4 \\
(17.4) \\
\end{array}$ & $\begin{array}{c}82.8 \\
(46.5) \\
\end{array}$ & $\begin{array}{c}80.4 \\
(100.0) \\
\end{array}$ & \multirow{2}{*}{1.203} & \multirow{2}{*}{4} \\
\hline & yes & $\begin{array}{c}21.1 \\
(6.3) \\
\end{array}$ & $\begin{array}{c}22.2 \\
(19.0)\end{array}$ & $\begin{array}{l}23.4 \\
(17.5) \\
\end{array}$ & $\begin{array}{c}19.6 \\
(17.5) \\
\end{array}$ & $\begin{array}{c}17.2 \\
(39.7) \\
\end{array}$ & $\begin{array}{c}19.6 \\
(100.0)\end{array}$ & & \\
\hline \multirow{2}{*}{$\begin{array}{l}\text { Take a } \\
\text { boat to the } \\
\text { Princes' } \\
\text { Islands } \\
p=0.683\end{array}$} & no & $\begin{array}{l}68.4 \\
(5.9) \\
\end{array}$ & $\begin{array}{c}74.1 \\
(18.1) \\
\end{array}$ & $\begin{array}{c}68.1 \\
(14.5) \\
\end{array}$ & $\begin{array}{c}74.5 \\
(18.5) \\
\end{array}$ & $\begin{array}{c}65.5 \\
(43.0) \\
\end{array}$ & $\begin{array}{c}69.1 \\
(100.0)\end{array}$ & \multirow[b]{2}{*}{2.286} & \multirow[b]{2}{*}{4} \\
\hline & yes & $\begin{array}{l}31.6 \\
(6.1)\end{array}$ & $\begin{array}{l}25.9 \\
(14.1)\end{array}$ & $\begin{array}{l}31.9 \\
(15.1)\end{array}$ & $\begin{array}{l}25.5 \\
(14.1)\end{array}$ & $\begin{array}{c}34.5 \\
(50.5)\end{array}$ & $\begin{array}{c}30.9 \\
(100.0)\end{array}$ & & \\
\hline \multirow{2}{*}{$\begin{array}{l}\text { Try the night } \\
\text { life } \\
p=0.380\end{array}$} & no & $\begin{array}{l}68.4 \\
(6.4) \\
\end{array}$ & $\begin{array}{c}66.7 \\
(17.7) \\
\end{array}$ & $\begin{array}{c}68.1 \\
(15.8) \\
\end{array}$ & $\begin{array}{c}69.6 \\
(19.2) \\
\end{array}$ & $\begin{array}{c}57.2 \\
(40.9) \\
\end{array}$ & $\begin{array}{c}63.2 \\
(100.0) \\
\end{array}$ & \multirow{2}{*}{4.199} & \multirow{2}{*}{4} \\
\hline & yes & $\begin{array}{l}31.6 \\
(5.1) \\
\end{array}$ & $\begin{array}{c}33.7 \\
(15.2) \\
\end{array}$ & $\begin{array}{c}31.9 \\
(12.7) \\
\end{array}$ & $\begin{array}{c}30.4 \\
(14.4) \\
\end{array}$ & $\begin{array}{c}42.8 \\
(52.5) \\
\end{array}$ & $\begin{array}{c}36.8 \\
(100.0)\end{array}$ & & \\
\hline
\end{tabular}

* Percent within variable (percent within cluster number of case); ** Statistically significant at $p<0.01$; *** Statistically significant at $p<0.05$

Table 7. Importance of different forms of retail outlets by clusters

\begin{tabular}{|c|c|c|c|c|c|c|c|c|c|}
\hline Variable & Description & $\mathrm{C} 1 *$ & $\mathrm{C} 2$ & C 3 & C 4 & $C 5$ & $\begin{array}{c}\text { Total } \\
\text { column }\end{array}$ & $\begin{array}{l}\text { Pearson's Chi- } \\
\text { square Value }\end{array}$ & Df \\
\hline \multirow{5}{*}{$\begin{array}{l}\text { Importance } \\
\text { of bazaars } \\
\text { (traditional } \\
\text { retail)** } \\
p=0.002\end{array}$} & 1 (Least important) & $\begin{array}{c}15.8 \\
(12.0)\end{array}$ & $\begin{array}{c}7.4 \\
(16.0)\end{array}$ & $\begin{array}{c}17.8 \\
(32.0)\end{array}$ & $\begin{array}{c}12.5 \\
(28.0)\end{array}$ & $\begin{array}{c}2.1 \\
(12.0)\end{array}$ & $\begin{array}{c}7.9 \\
(100.0)\end{array}$ & \multirow{5}{*}{37.719} & \multirow{5}{*}{16} \\
\hline & 2 & $\begin{array}{l}15.8 \\
(8.6)\end{array}$ & $\begin{array}{c}5.5 \\
(8.6)\end{array}$ & $\begin{array}{c}15.5 \\
(20.0)\end{array}$ & $\begin{array}{c}19.6 \\
(31.4)\end{array}$ & $\begin{array}{c}7.6 \\
(31.4)\end{array}$ & $\begin{array}{c}11.0 \\
(100.0)\end{array}$ & & \\
\hline & 3 & $\begin{array}{c}31.6 \\
(10.2)\end{array}$ & $\begin{array}{c}25.9 \\
(23.7)\end{array}$ & $\begin{array}{c}15.5 \\
(11.9)\end{array}$ & $\begin{array}{c}16.1 \\
(15.2)\end{array}$ & $\begin{array}{c}16.0 \\
(40.0)\end{array}$ & $\begin{array}{c}18.5 \\
(100.0)\end{array}$ & & \\
\hline & 4 & $\begin{array}{l}10.5 \\
(1.7) \\
\end{array}$ & $\begin{array}{c}33.3 \\
(15.4) \\
\end{array}$ & $\begin{array}{c}31.1 \\
(12.0) \\
\end{array}$ & $\begin{array}{c}26.8 \\
(12.8) \\
\end{array}$ & $\begin{array}{c}47.2 \\
(58.1) \\
\end{array}$ & $\begin{array}{c}36.8 \\
(100.0)\end{array}$ & & \\
\hline & 5 (most important & $\begin{array}{l}26.3 \\
(6.1)\end{array}$ & $\begin{array}{c}27.7 \\
(18.3)\end{array}$ & $\begin{array}{l}20.0 \\
(11.0)\end{array}$ & $\begin{array}{l}25.0 \\
(17.1)\end{array}$ & $\begin{array}{c}27.1 \\
(47.6)\end{array}$ & $\begin{array}{c}25.8 \\
(100.0)\end{array}$ & & \\
\hline \multirow{5}{*}{$\begin{array}{l}\text { Importance } \\
\text { of malls } \\
\text { (modern } \\
\text { retail)** } \\
p=0.000\end{array}$} & 1 (least important) & $\begin{array}{l}10.5 \\
(2.7)\end{array}$ & $\begin{array}{c}40.4 \\
(28.0)\end{array}$ & $\begin{array}{c}25.0 \\
(14.7)\end{array}$ & $\begin{array}{c}22.2 \\
(16.0)\end{array}$ & $\begin{array}{c}20.4 \\
(38.7)\end{array}$ & $\begin{array}{c}24.1 \\
(100.0)\end{array}$ & \multirow{5}{*}{48.196} & \multirow{5}{*}{16} \\
\hline & 2 & $\begin{array}{c}0.0 \\
(0.0)\end{array}$ & $\begin{array}{l}11.5 \\
(8.8) \\
\end{array}$ & $\begin{array}{l}29.5 \\
(19.1)\end{array}$ & $\begin{array}{c}25.9 \\
(20.6)\end{array}$ & $\begin{array}{c}24.6 \\
(51.5)\end{array}$ & $\begin{array}{c}31.9 \\
(100.0)\end{array}$ & & \\
\hline & 3 & $\begin{array}{l}31.6 \\
(6.6)\end{array}$ & $\begin{array}{l}28.8 \\
(16.5)\end{array}$ & $\begin{array}{c}31.8 \\
(15.4)\end{array}$ & $\begin{array}{c}27.7 \\
(16.5)\end{array}$ & $\begin{array}{l}28.8 \\
(45.1)\end{array}$ & $\begin{array}{c}29.3 \\
(100.0)\end{array}$ & & \\
\hline & 4 & $\begin{array}{l}15.8 \\
(5.9)\end{array}$ & $\begin{array}{c}11.5 \\
(11.8)\end{array}$ & $\begin{array}{c}9.1 \\
(7.8)\end{array}$ & $\begin{array}{c}18.5 \\
(19.6)\end{array}$ & $\begin{array}{c}19.7 \\
(54.9)\end{array}$ & $\begin{array}{c}16.4 \\
(100.0)\end{array}$ & & \\
\hline & 5 (most important) & $\begin{array}{c}42.1 \\
(30.8)\end{array}$ & $\begin{array}{c}7.7 \\
(15.4)\end{array}$ & $\begin{array}{c}4.5 \\
(7.7)\end{array}$ & $\begin{array}{c}5.5 \\
(11.5)\end{array}$ & $\begin{array}{c}6.3 \\
(34.6)\end{array}$ & $\begin{array}{c}8.4 \\
(100.0)\end{array}$ & & \\
\hline
\end{tabular}




\begin{tabular}{|c|c|c|c|c|c|c|c|c|c|}
\hline Variable & Description & C $1 *$ & $C 2$ & C 3 & C 4 & C 5 & $\begin{array}{c}\text { Total } \\
\text { column }\end{array}$ & $\begin{array}{l}\text { Pearson's Chi- } \\
\text { square Value }\end{array}$ & Df \\
\hline \multirow{5}{*}{$\begin{array}{l}\text { Importance } \\
\text { of arts } \\
\text { and crafts } \\
\text { centers** } \\
p=0.003\end{array}$} & 1 (least important) & $\begin{array}{l}23.5 \\
(12.1)\end{array}$ & $\begin{array}{l}3.8 \\
(6.1)\end{array}$ & $\begin{array}{c}25.0 \\
(33.3)\end{array}$ & $\begin{array}{c}14.3 \\
(24.2)\end{array}$ & $\begin{array}{c}5.6 \\
(24.2)\end{array}$ & $\begin{array}{c}10.5 \\
(100.0)\end{array}$ & \multirow{5}{*}{35.719} & \multirow{2}{*}{16} \\
\hline & 2 & $\begin{array}{c}11.8 \\
(6.25) \\
\end{array}$ & $\begin{array}{c}15.1 \\
(25.0) \\
\end{array}$ & $\begin{array}{c}15.9 \\
(21.9)\end{array}$ & $\begin{array}{c}3.6 \\
(6.25) \\
\end{array}$ & $\begin{array}{c}9.0 \\
(40.6)\end{array}$ & $\begin{array}{c}10.2 \\
(100.0)\end{array}$ & & \\
\hline & 3 & $\begin{array}{l}29.4 \\
(5.6) \\
\end{array}$ & $\begin{array}{c}34.0 \\
(20.2)\end{array}$ & $\begin{array}{c}31.8 \\
(15.7) \\
\end{array}$ & $\begin{array}{c}30.4 \\
(19.1) \\
\end{array}$ & $\begin{array}{c}24.3 \\
(39.3) \\
\end{array}$ & $\begin{array}{c}28.3 \\
(100.0)\end{array}$ & & \\
\hline & 4 & $\begin{array}{l}29.4 \\
(4.6) \\
\end{array}$ & $\begin{array}{c}28.3 \\
(13.8) \\
\end{array}$ & $\begin{array}{l}20.4 \\
(8.3) \\
\end{array}$ & $\begin{array}{c}32.1 \\
(16.5)\end{array}$ & $\begin{array}{c}43.1 \\
(59.0) \\
\end{array}$ & $\begin{array}{c}34.7 \\
(100.0)\end{array}$ & & \\
\hline & 5 (most important) & $\begin{array}{c}5.9 \\
(2.0) \\
\end{array}$ & $\begin{array}{c}18.9 \\
(19.6)\end{array}$ & $\begin{array}{c}6.8 \\
(5.9) \\
\end{array}$ & $\begin{array}{c}19.6 \\
(21.6) \\
\end{array}$ & $\begin{array}{c}18.0 \\
(51.0)\end{array}$ & $\begin{array}{c}16.2 \\
(100.0) \\
\end{array}$ & & \\
\hline \multirow{5}{*}{$\begin{array}{l}\text { Importance } \\
\text { of airport } \\
\text { outlets } \\
p=0.814\end{array}$} & 1 (least important) & $\begin{array}{l}15.8 \\
(3.2) \\
\end{array}$ & $\begin{array}{c}30.2 \\
(17.0)\end{array}$ & $\begin{array}{l}40.0 \\
(19.1) \\
\end{array}$ & $\begin{array}{c}21.8 \\
(12.8) \\
\end{array}$ & $\begin{array}{c}31.7 \\
(47.9) \\
\end{array}$ & $\begin{array}{c}29.9 \\
(100.0)\end{array}$ & \multirow{5}{*}{10.933} & \multirow{5}{*}{16} \\
\hline & 2 & $\begin{array}{l}21.0 \\
(5.4) \\
\end{array}$ & $\begin{array}{c}20.7 \\
(14.9) \\
\end{array}$ & $\begin{array}{l}20.0 \\
(12.2) \\
\end{array}$ & $\begin{array}{c}25.4 \\
(18.9) \\
\end{array}$ & $\begin{array}{c}25.3 \\
(48.6) \\
\end{array}$ & $\begin{array}{c}23.6 \\
(100.0) \\
\end{array}$ & & \\
\hline & 3 & $\begin{array}{c}35.8 \\
(10.8) \\
\end{array}$ & $\begin{array}{c}18.9 \\
(15.4) \\
\end{array}$ & $\begin{array}{c}17.8 \\
(12.3) \\
\end{array}$ & $\begin{array}{l}23.6 \\
(20.0) \\
\end{array}$ & $\begin{array}{c}19.0 \\
(41.5)\end{array}$ & $\begin{array}{c}20.7 \\
(100.0) \\
\end{array}$ & & \\
\hline & 4 & $\begin{array}{l}10.5 \\
(4.4) \\
\end{array}$ & $\begin{array}{c}18.9 \\
(22.2) \\
\end{array}$ & $\begin{array}{c}15.6 \\
(15.6)\end{array}$ & $\begin{array}{c}14.5 \\
(17.8)\end{array}$ & $\begin{array}{c}12.7 \\
(40.0)\end{array}$ & $\begin{array}{c}14.3 \\
(100.0)\end{array}$ & & \\
\hline & 5 (most important) & $\begin{array}{l}15.8 \\
(8.3) \\
\end{array}$ & $\begin{array}{c}11.3 \\
(16.7) \\
\end{array}$ & $\begin{array}{c}6.7 \\
(8.3) \\
\end{array}$ & $\begin{array}{c}14.5 \\
(22.2) \\
\end{array}$ & $\begin{array}{c}11.3 \\
(44.4) \\
\end{array}$ & $\begin{array}{c}11.5 \\
(100.0) \\
\end{array}$ & & \\
\hline \multirow{5}{*}{$\begin{array}{l}\text { Importance } \\
\text { or wholesale } \\
p=0.053\end{array}$} & 1 (least important) & $\begin{array}{l}23.5 \\
(5.2) \\
\end{array}$ & $\begin{array}{c}19.6 \\
(13.0)\end{array}$ & $\begin{array}{c}31.8 \\
(18.2)\end{array}$ & $\begin{array}{c}25.4 \\
(18.2)\end{array}$ & $\begin{array}{l}24.8 \\
(45.4) \\
\end{array}$ & $\begin{array}{c}25.0 \\
(100.0)\end{array}$ & \multirow{5}{*}{26.073} & \multirow{5}{*}{16} \\
\hline & 2 & $\begin{array}{l}11.8 \\
(2.6) \\
\end{array}$ & $\begin{array}{l}33.3 \\
(9.0) \\
\end{array}$ & $\begin{array}{c}27.3 \\
(15.4) \\
\end{array}$ & $\begin{array}{c}23.6 \\
(16.7) \\
\end{array}$ & $\begin{array}{c}24.1 \\
(43.6) \\
\end{array}$ & $\begin{array}{c}25.3 \\
(100.0)\end{array}$ & & \\
\hline & 3 & $\begin{array}{l}23.5 \\
(4.9) \\
\end{array}$ & $\begin{array}{c}23.5 \\
(14.6) \\
\end{array}$ & $\begin{array}{l}18.2 \\
(9.8) \\
\end{array}$ & $\begin{array}{c}30.9 \\
(20.7) \\
\end{array}$ & $\begin{array}{c}29.1 \\
(50.0) \\
\end{array}$ & $\begin{array}{c}26.6 \\
(100.0)\end{array}$ & & \\
\hline & 4 & $\begin{array}{l}11.8 \\
(3.8) \\
\end{array}$ & $\begin{array}{c}17.6 \\
(17.3) \\
\end{array}$ & $\begin{array}{c}22.7 \\
(19.2) \\
\end{array}$ & $\begin{array}{c}12.7 \\
(13.5) \\
\end{array}$ & $\begin{array}{c}17.0 \\
(46.1)\end{array}$ & $\begin{array}{c}16.9 \\
(100.0)\end{array}$ & & \\
\hline & 5 (most important) & $\begin{array}{c}29.4 \\
(26.3)\end{array}$ & $\begin{array}{c}5.9 \\
(15.8)\end{array}$ & $\begin{array}{l}0.0 \\
(0.0)\end{array}$ & $\begin{array}{c}7.3 \\
(21.0)\end{array}$ & $\begin{array}{c}5.0 \\
(36.8)\end{array}$ & $\begin{array}{c}6.2 \\
(100.0)\end{array}$ & & \\
\hline
\end{tabular}

* Percent within variable (percent within cluster number of case); ** Statistically significant at $p<0.01$

Table 8. Tourists' perception of shopping benefits.

\begin{tabular}{|c|c|c|c|c|c|c|c|c|c|}
\hline Variable & Description & $\mathrm{C} 1^{*}$ & $C 2$ & C 3 & C 4 & $C 5$ & $\begin{array}{c}\text { Total } \\
\text { column }\end{array}$ & $\begin{array}{l}\text { Pearson's Chi- } \\
\text { square Value }\end{array}$ & Df \\
\hline \multirow{5}{*}{$\begin{array}{l}\text { It relaxes } \\
\text { me** } \\
p=0.005\end{array}$} & 1 (least important) & $\begin{array}{l}35.3 \\
(9.4)\end{array}$ & $\begin{array}{c}27.4 \\
(21.9)\end{array}$ & $\begin{array}{c}35.5 \\
(25.0)\end{array}$ & $\begin{array}{l}23.6 \\
(20.3)\end{array}$ & $\begin{array}{c}10.7 \\
(23.4)\end{array}$ & $\begin{array}{c}20.8 \\
(100.0)\end{array}$ & \multirow{5}{*}{34.313} & \multirow{5}{*}{16} \\
\hline & 2 & $\begin{array}{l}23.5 \\
(8.7)\end{array}$ & $\begin{array}{c}11.8 \\
(13.0)\end{array}$ & $\begin{array}{l}8.9 \\
(8.7)\end{array}$ & $\begin{array}{c}18.2 \\
(21.7)\end{array}$ & $\begin{array}{c}15.7 \\
(47.8)\end{array}$ & $\begin{array}{c}14.9 \\
(100.0)\end{array}$ & & \\
\hline & 3 & $\begin{array}{c}0.0 \\
(0.0)\end{array}$ & $\begin{array}{c}31.4 \\
(21.9) \\
\end{array}$ & $\begin{array}{l}24.4 \\
(15.1) \\
\end{array}$ & $\begin{array}{c}16.4 \\
(12.3) \\
\end{array}$ & $\begin{array}{c}26.4 \\
(50.7) \\
\end{array}$ & $\begin{array}{c}23.7 \\
(100.0)\end{array}$ & & \\
\hline & 4 & $\begin{array}{l}11.8 \\
(2.4)\end{array}$ & $\begin{array}{l}21.6 \\
(13.2)\end{array}$ & $\begin{array}{l}20.0 \\
(10.8)\end{array}$ & $\begin{array}{c}25.4 \\
(18.9)\end{array}$ & $\begin{array}{c}33.6 \\
(56.6)\end{array}$ & $\begin{array}{c}26.9 \\
(100.0)\end{array}$ & & \\
\hline & 5 (most important) & $\begin{array}{l}29.4 \\
(11.9)\end{array}$ & $\begin{array}{l}7.8 \\
(9.5)\end{array}$ & $\begin{array}{c}11.1 \\
(11.9)\end{array}$ & $\begin{array}{c}16.4 \\
(21.4)\end{array}$ & $\begin{array}{c}13.6 \\
(45.2)\end{array}$ & $\begin{array}{c}13.6 \\
(100.0)\end{array}$ & & \\
\hline
\end{tabular}




\begin{tabular}{|c|c|c|c|c|c|c|c|c|c|}
\hline Variable & Description & $C 1^{*}$ & $C 2$ & C 3 & C 4 & $C 5$ & $\begin{array}{c}\text { Total } \\
\text { column }\end{array}$ & $\begin{array}{l}\text { Pearson's Chi- } \\
\text { square Value }\end{array}$ & Df \\
\hline \multirow{5}{*}{$\begin{array}{l}\text { It is an } \\
\text { opportunity } \\
\text { to escape the } \\
\text { routine** } \\
p=0.002\end{array}$} & 1 (least important) & $\begin{array}{l}29.4 \\
(9.4) \\
\end{array}$ & $\begin{array}{c}17.6 \\
(17.0) \\
\end{array}$ & $\begin{array}{l}38.6 \\
(32.1) \\
\end{array}$ & $\begin{array}{l}22.6 \\
(22.6) \\
\end{array}$ & $\begin{array}{c}7.2 \\
(18.9) \\
\end{array}$ & $\begin{array}{c}17.5 \\
(100.0) \\
\end{array}$ & \multirow{5}{*}{36.500} & \multirow{5}{*}{16} \\
\hline & 2 & $\begin{array}{l}17.6 \\
(6.4) \\
\end{array}$ & $\begin{array}{c}17.6 \\
(19.1) \\
\end{array}$ & $\begin{array}{c}9.1 \\
(8.5) \\
\end{array}$ & $\begin{array}{c}15.1 \\
(17.0) \\
\end{array}$ & $\begin{array}{c}16.7 \\
(48.9) \\
\end{array}$ & $\begin{array}{c}15.5 \\
(100.0) \\
\end{array}$ & & \\
\hline & 3 & $\begin{array}{l}17.6 \\
(3.9) \\
\end{array}$ & $\begin{array}{l}23.5 \\
(15.6) \\
\end{array}$ & $\begin{array}{l}20.4 \\
(11.7) \\
\end{array}$ & $\begin{array}{l}20.8 \\
(14.3) \\
\end{array}$ & $\begin{array}{c}30.4 \\
(54.5) \\
\end{array}$ & $\begin{array}{c}25.4 \\
(100.0)\end{array}$ & & \\
\hline & 4 & $\begin{array}{l}11.8 \\
(2.1) \\
\end{array}$ & $\begin{array}{c}35.3 \\
(18.8) \\
\end{array}$ & $\begin{array}{c}27.3 \\
(12.5) \\
\end{array}$ & $\begin{array}{c}35.8 \\
(19.8) \\
\end{array}$ & $\begin{array}{c}32.6 \\
(46.9) \\
\end{array}$ & $\begin{array}{c}31.7 \\
(100.0)\end{array}$ & & \\
\hline & 5 (most important) & $\begin{array}{l}23.5 \\
(13.3) \\
\end{array}$ & $\begin{array}{c}5.9 \\
(10.0) \\
\end{array}$ & $\begin{array}{c}4.5 \\
(6.7) \\
\end{array}$ & $\begin{array}{c}5.7 \\
(10.0) \\
\end{array}$ & $\begin{array}{c}13.0 \\
(60.0)\end{array}$ & $\begin{array}{c}9.9 \\
(100.0)\end{array}$ & & \\
\hline \multirow{5}{*}{$\begin{array}{l}\text { It is a social } \\
\text { opportu- } \\
\text { nity*** } \\
p=0.012\end{array}$} & 1 (least important) & $\begin{array}{l}29.4 \\
(11.6) \\
\end{array}$ & $\begin{array}{c}12.0 \\
(13.9)\end{array}$ & $\begin{array}{c}31.8 \\
(32.6) \\
\end{array}$ & $\begin{array}{c}9.4 \\
(11.6) \\
\end{array}$ & $\begin{array}{c}9.3 \\
(30.2)\end{array}$ & $\begin{array}{c}14.1 \\
(100.0)\end{array}$ & \multirow{5}{*}{31.380} & \multirow{5}{*}{16} \\
\hline & 2 & $\begin{array}{l}23.5 \\
(7.8) \\
\end{array}$ & $\begin{array}{c}12.0 \\
(11.8) \\
\end{array}$ & $\begin{array}{c}15.9 \\
(13.7) \\
\end{array}$ & $\begin{array}{c}17.0 \\
(17.6)\end{array}$ & $\begin{array}{c}17.9 \\
(49.0) \\
\end{array}$ & $\begin{array}{c}16.8 \\
(100.0)\end{array}$ & & \\
\hline & 3 & $\begin{array}{l}17.6 \\
(3.2) \\
\end{array}$ & $\begin{array}{l}34.0 \\
(17.9)\end{array}$ & $\begin{array}{c}36.4 \\
(16.8)\end{array}$ & $\begin{array}{l}22.6 \\
(12.6)\end{array}$ & $\begin{array}{c}33.6 \\
(49.5) \\
\end{array}$ & $\begin{array}{c}31.3 \\
(100.0)\end{array}$ & & \\
\hline & 4 & $\begin{array}{l}17.6 \\
(3.3) \\
\end{array}$ & $\begin{array}{c}36.0 \\
(19.8)\end{array}$ & $\begin{array}{l}11.4 \\
(5.5) \\
\end{array}$ & $\begin{array}{c}43.4 \\
(25.3) \\
\end{array}$ & $\begin{array}{c}30.0 \\
(46.1)\end{array}$ & $\begin{array}{c}29.9 \\
(100.0)\end{array}$ & & \\
\hline & 5 (most important) & $\begin{array}{l}11.8 \\
(8.3)\end{array}$ & $\begin{array}{c}6.0 \\
(12.5)\end{array}$ & $\begin{array}{c}4.5 \\
(8.3)\end{array}$ & $\begin{array}{c}7.5 \\
(16.7)\end{array}$ & $\begin{array}{c}9.3 \\
(54.2)\end{array}$ & $\begin{array}{c}7.9 \\
(100.0)\end{array}$ & & \\
\hline \multirow{5}{*}{$\begin{array}{l}\text { I don't like } \\
\text { shopping*** } \\
p=0.013\end{array}$} & 1 (least important) & $\begin{array}{c}58.8 \\
(10.7) \\
\end{array}$ & $\begin{array}{l}26.9 \\
(15.0)\end{array}$ & $\begin{array}{c}31.8 \\
(15.0)\end{array}$ & $\begin{array}{l}24.5 \\
(14.0)\end{array}$ & $\begin{array}{c}30.0 \\
(45.2)\end{array}$ & $\begin{array}{c}30.4 \\
(100.0)\end{array}$ & \multirow{5}{*}{31.241} & \multirow{5}{*}{16} \\
\hline & 2 & $\begin{array}{l}11.8 \\
(2.7) \\
\end{array}$ & $\begin{array}{c}17.3 \\
(12.0) \\
\end{array}$ & $\begin{array}{l}11.4 \\
(6.7) \\
\end{array}$ & $\begin{array}{c}34.0 \\
(24.0) \\
\end{array}$ & $\begin{array}{c}29.3 \\
(54.7) \\
\end{array}$ & $\begin{array}{c}24.5 \\
(100.0)\end{array}$ & & \\
\hline & 3 & $\begin{array}{c}0.0 \\
(0.0)\end{array}$ & $\begin{array}{c}15.4 \\
(17.8)\end{array}$ & $\begin{array}{c}22.7 \\
(22.2)\end{array}$ & $\begin{array}{c}11.3 \\
(13.3)\end{array}$ & $\begin{array}{c}15.0 \\
(46.7)\end{array}$ & $\begin{array}{c}14.7 \\
(100.0)\end{array}$ & & \\
\hline & 4 & $\begin{array}{l}17.6 \\
(5.7) \\
\end{array}$ & $\begin{array}{c}23.1 \\
(22.6) \\
\end{array}$ & $\begin{array}{c}9.1 \\
(7.5) \\
\end{array}$ & $\begin{array}{c}15.1 \\
(15.1) \\
\end{array}$ & $\begin{array}{c}18.6 \\
(46.1)\end{array}$ & $\begin{array}{c}17.3 \\
(100.0)\end{array}$ & & \\
\hline & 5 (most important) & $\begin{array}{l}11.8 \\
(5.0)\end{array}$ & $\begin{array}{c}17.3 \\
(22.5)\end{array}$ & $\begin{array}{l}25.0 \\
(27.5)\end{array}$ & $\begin{array}{c}15.1 \\
(20.0)\end{array}$ & $\begin{array}{c}7.1 \\
825.0)\end{array}$ & $\begin{array}{c}13.1 \\
(100.0)\end{array}$ & & \\
\hline
\end{tabular}

* Percent within variable (percent within cluster number of case); ** Statistically significant at $p<0.01$; ** Statistically significant at $p<0.05$

ly significant differences between tourist market segments we did not find evidence to support such claims (table 4).

In terms of tourists' travel characteristics (table 5), we note statistically significant differences between clusters based on the number of persons travelling together, the number of previous visits to Istanbul and length of stay. Non-significant differences were found for means of travel and type of accommodation. The results of the present research support some previous finding while contradicting others (table 9). Our study revealed that the majority of 'product-focused shoppers' travels alone. A significant minority of lone travellers is found also among 'difference seekers'. Most of the tourists in the other clusters travel in small groups of up to four persons with either family or friends. We also found that a higher proportion of tourists who travel in larger groups are included in the reluctant shoppers' category. While four segments are dominated by tourists who have never visited Istanbul in the past, more than one-third (the largest group) of 'prod- uct-focused shoppers' has been many times to the city. Also, while spending 3-7 nights seems to be the most likely length of stay in all five clusters, we should note that the highest proportion of short-time visitors (less than 24 hours) is among 'product-focused shoppers'. In addition, when looking at 'reluctant shoppers' we conclude that tourists in this group also tend to favour shorter stays. Thus, regarding this group, we observe that a much larger percentage than average choose to spend only 1-2 nights in Istanbul while a smaller percentage than average in the same group spend more than a week.

Where motivation to travel to Istanbul and travel activities are concerned (table 6), we found statistically significant differences between groups based on primary motivation. Thus, we found that pleasure tourists dominate four of the five clusters. The exception is cluster 1 ('product-focused shoppers') where the proportion of tourists travelling for business and those travelling for pleasure is equal (36.8\%). This cluster also includes a large proportion of tourists in transit 
Table 9. The importance of travel characteristics in profiling tourist clusters in previous studies

\begin{tabular}{|l|c|c|}
\hline \multirow{2}{*}{ Travel characteristics } & \multicolumn{2}{|c|}{ Generate statistically significant differences among clusters } \\
\cline { 2 - 3 } & \multicolumn{1}{|c|}{ Nes } \\
\hline Number of persons travelling & Carmichael, Smith (2004); D'Urso et al. (2016) & Mody et al. (2014); Tkaczynski et al. (2009) \\
\hline Means of travel & Carmichael, Smith (2004) & Andreu et al. (2005) \\
\hline Accommodation choice & $\begin{array}{c}\text { Andreu et al. (2005); D'Urso et al. (2016); Mody } \\
\text { et al. (2014); Park, Yoon (2009); Rid et al. (2014) }\end{array}$ & D'Urso et al. (2013) \\
\hline Number of previous visits & $\begin{array}{c}\text { Andreu et al. (2005); Brida et al. (2014); } \\
\text { CarmichaelSmith (2014) }\end{array}$ & Mody et al. (2014) \\
\hline
\end{tabular}

(26.8\%) and does not include anyone visiting to meet with family and/or friends. We also found that more than half $(57 \%)$ of those tourists in the 'product-focused shoppers' category who declared pleasure as their primary motive to visit Istanbul also listed 'shopping' as their main reason for travelling there. On the other hand, only between 8 and 14 percent of pleasure tourists in the other four categories travelled to Istanbul for shopping. Half of the 'difference-seekers' and a little under half (47.2\%) of the 'shoppers for cultural experience' visited the city mainly to experience a new culture while almost $56 \%$ of the 'reluctant shoppers' and more than $53 \%$ of the 'total shoppers' who classified themselves as 'pleasure tourists' decided to visit Istanbul for its historical and/or cultural sites. Our results resonate with previous findings by Andreu et al. (2005), Carmichael, Smith (2004), D’Urso et al. (2016) and Lopez-Guzman et al. (2014) and are contrary to findings by $\mathrm{Hu}, \mathrm{Yu}(2007)$.

Our findings also confirm claims by Lopez-Guzman et al. (2014) and Park, Yoon (2009) that tourist activities at the destination can be linked to the visitors' motivation to shop. We found that three of the activities in cluded in the survey produced statistically significant differences between clusters: 'visiting historical/cultural sites', 'taking a tour of the Bosporus' and 'attending cultural events' (table 6). The study reveals that the most likely to visit historical/cultural sites are travellers who belong to clusters 2 ('shoppers for cultural experience') and 5 ('total shoppers') while least likely were 'product-focused shoppers' and 'reluctant shoppers' (although more than half of them have visited or wanted to visit these sites). 'Total shoppers' were also the most likely tourists to take a tour on the Bosporus while 'difference-seekers' were the only group in which less than half of the members were interested in these tours. Furthermore, not surprisingly, 'shoppers for cultural experience' were the most interested in attending cultural events (although less than half expressed intent) while, surprisingly, the least inclined to attend these events were the 'difference seekers'.

In terms of perceived importance of different types of outlets (table 7) we found significant differences be- tween clusters based on the importance assigned to traditional retail outlets (bazaars), malls and arts and crafts stores while preference for airport outlets or wholesale stores did not reveal any significant differences. We found that 'product-focused shoppers' were the least interested in bazaars while 'total shoppers' and 'shoppers for cultural experience' were the most interested. On the other hand, 'product-focused shoppers' were the most likely to see malls as important while 'reluctant shoppers' and 'shoppers for cultural difference' were the least likely. Finally, the arts and crafts concept stores presented the most interest to 'total shoppers' and 'difference seekers' while 'reluctant shoppers' and 'product-focused shoppers' were the least interested. Our findings are very important because few other studies have considered shopping outlet choice to be an important indicator to describe the composition of different shopping tourism segments (for example, the study by $\mathrm{Hu}, \mathrm{Yu}, 2007$ ).

Finally, we were interested in how travellers in the five clusters perceived the potential benefits of shopping. We found that all four statements generated statistically significant differences (table 8). The results reveal that 'total shoppers' are the most likely to agree with the statement that shopping helps with relaxation while 'shoppers for cultural experience' seem to be the least likely to agree with the statement. Interesting to mention here is also that 'product-focused shoppers' are split between those who agree and those who disagree with the statement. While this situation may be interesting it certainly is not surprising since an important percentage of tourists in this category travel to Istanbul for business and not for leisure; therefore, for these tourists, shopping is part of their work and not a relaxing activity. 'Total shoppers' were also the most likely to agree with the statement that shopping is an opportunity for them to escape the routine while 'reluctant shoppers' and 'product-focused shoppers' were the least likely to agree. Travellers in these two groups were also the least likely to agree with the statement that shopping provides an opportunity to interact with other people. 'Difference-seekers' were found to be the most likely to perceive shopping as 
a social opportunity. Finally, not surprisingly, 'reluctant shoppers' were found to be the most likely to dislike shopping while 'total shoppers' were the least likely. Again, 'product-focused shoppers' were somewhat split on this idea.

\section{Characteristics of tourists in the five clusters}

The main characteristics of the members of the five clusters are detailed in table 10 below:

The table above shows interesting differences among the five clusters. Thus, while product-focused shoppers travel to Istanbul mainly from Africa and the Middle East, the majority of tourist shoppers in the other clusters are European. Product-focused shoppers travel alone, visit Istanbul often (normally for short periods of time and, perhaps, shop as part of their business. They buy merchandise in Istanbul and re-sell it in their home country. For this reason, they prefer malls where they can find global and Turkish brands at competitive prices.

Shoppers for cultural experience are generally firsttimers. They travel to Istanbul mainly to visit historical/cultural sites and to attend cultural events. They are the least likely to feel relaxed when shopping and when they do shop they prefer bazaar (perhaps because they perceive shopping in bazaar as a cultural experience).

Reluctant shoppers are the most likely to dislike shopping. They are, also, the least likely to perceive shopping as an opportunity to escape routine of to meet others. Thus, this type of tourist shoppers is the least interested in malls and arts and crafts centres. Generally speaking these tourists are less interested than any other group in visiting any type of shopping centre.

Difference-seekers are mainly pleasure tourists interested in cultural differences. They perceive shopping as an opportunity to meet other people and like to shop in arts and crafts stores.

Total shoppers are pleasure tourists, visiting Istanbul for the first time. They like to visit historical/cultural sites and take a tour of the Bosporus. They also like shopping because relaxes them and helps them break the routine. They shop in all types of shopping outlets but prefer bazaars and arts and crafts centres.

The table above demonstrates that the resulting five segments are quite different from each other in terms of socio-demographic and travel characteristics

Table 10. Main characteristics of tourists in the five clusters

\begin{tabular}{|c|c|c|c|c|}
\hline $\begin{array}{l}\text { Product-focused } \\
\text { shoppers }\end{array}$ & $\begin{array}{l}\text { Shoppers for cultural } \\
\text { experience }\end{array}$ & Reluctant shoppers & Difference seekers & Total shoppers \\
\hline $\begin{array}{l}\text { - coming from the } \\
\text { Middle East and } \\
\text { North Africa and } \\
\text { from Sub-Saharan } \\
\text { Africa } \\
\text { - lone visitors } \\
\text { - multiple visits } \\
\text { - very short visits } \\
\text { - visiting for business/ } \\
\text { pleasure } \\
\text { - many in transit } \\
\text { - not visiting to meet } \\
\text { with family or friends } \\
\text { - less likely to visit } \\
\text { historical/cultural } \\
\text { sites } \\
\text { - like to shop in malls } \\
\text { - least interested in } \\
\text { bazaars and arts and } \\
\text { crafts stores } \\
\text { - shopping relaxes } \\
\text { some } \\
\text { - least likely to } \\
\text { perceive shopping } \\
\text { as an opportunity to } \\
\text { escape the routine or } \\
\text { as an opportunity to } \\
\text { meet others } \\
\text { - some like while } \\
\text { others dislike } \\
\text { shopping }\end{array}$ & $\begin{array}{l}\text { - mainly coming from } \\
\text { - trave in small groups } \\
\text { ( } 2-4 \text { persons) with } \\
\text { family or friends } \\
\text { - first time in Istanbul } \\
\text { - pleasure tourists } \\
\text { - visit historical/ } \\
\text { cultural sites } \\
\text { - attend cultural } \\
\text { events } \\
\text { - like to shop in } \\
\text { bazaars } \\
\text { - least interested in } \\
\text { malls } \\
\text { - least likely to feel } \\
\text { relaxed while } \\
\text { shopping }\end{array}$ & $\begin{array}{l}\text { - } \text { mainly Europeans } \\
\text { - travel in small } \\
\text { groups (2-4 persons) } \\
\text { - some travel in large } \\
\text { groups (over } 4 \\
\text { persons) } \\
\text { - first time in Istanbul } \\
\text { - shorter visits } \\
\text { - pleasure tourists } \\
\text { - less likely to visit } \\
\text { historical/ } \\
\text { - cultural sites } \\
\text { - least interested in } \\
\text { malls and arts and } \\
\text { crafts centres } \\
\text { - least likely to } \\
\text { perceive shopping } \\
\text { as an opportunity to } \\
\text { escape routine or as } \\
\text { an opportunity to } \\
\text { meet others } \\
\text { - most likely to dislike } \\
\text { shopping }\end{array}$ & $\begin{array}{l}\text { - travel in small groups } \\
\text { (2-4 persons) with } \\
\text { family or friends } \\
\text { - some lone visitors } \\
\text { - pleasure tourists } \\
\text { - least likely to take a } \\
\text { tour of the Bosporus } \\
\text { and least likely to } \\
\text { attend cultural } \\
\text { events } \\
\text { - like to shop in arts } \\
\text { and crafts stores } \\
\text { - perceive shopping } \\
\text { as an opportunity to } \\
\text { meet other people }\end{array}$ & $\begin{array}{l}\text { - } \text { mainly Europeans } \\
\text { - travel in small groups } \\
\text { (2-4 persons) with } \\
\text { - family or friends } \\
\text { - } \text { first time in Istanbul } \\
\text { - visit historical/ } \\
\text { cultural sites } \\
\text { - take a tour of the } \\
\text { Bosporus } \\
\text { - like to shop in } \\
\text { bazaars and arts and } \\
\text { crafts stores } \\
\text { - shopping relaxes } \\
\text { them } \\
\text { - shop to escape the } \\
\text { routine }\end{array}$ \\
\hline
\end{tabular}


of tourists, their perception of shopping, their preference for certain types of shopping outlets and their interest in other activities while visiting Istanbul. This information could be used by marketers to target the right kind of tourist for each type of shopping outlet in Istanbul.

\section{Conclusion}

In this study,the researcher used market segmentation to learn more about the motivations of tourists to shop while visiting Istanbul. $\mathrm{He}$, then, used the resulting market typologies to better understand visitors' choices of shopping venues as well as their preferences and needs (Andreu, et al., 2005).

\section{Theoretical and Managerial Implications}

The findings have demonstrated that, while tourists' motivation to shop is multidimensional, travellers to a destination can be divided into more homogeneous groups based on the main reason they engage in shopping. The study found five types of tourists based on their motivation to shop while travelling: product-focused shoppers, shoppers for cultural experience, reluctant shoppers, difference seekers and total shoppers, each of these typologies being characterized by a different set of demographics, travel characteristics, shopping preferences and attitudes towards shopping. These groups were shown to differ significantly on a number of variables.

While this study is not the first to segment tourists based on their motivation to visit a destination, it is novel in that it deals with tourist shoppers and in that it focuses on a geographical area (Turkey and the Middle East) that has been generally ignored or not treated with the importance it deserves in the tourism literature. Furthermore, most studies are dealing with certain segments of tourist shoppers, such as souvenir shoppers (Hu,Yu, 2007; Swanson, Horridge, 2006), rural tourist shoppers (Carmichael, Smith, 2004), traditional market shoppers (Hsieh, Chang, 2006), mall shoppers (Le Hew, Wesley, 2007), shopping village shoppers (Murphy, et al., 2011), luxury shoppers (Park, et al., 2010) or shoppers from a certain country or region (Chen, et al., 2014; Mak, et al., 1999; Mok, Iverson, 2000) and were conducted in the proximity of different shopping venues. Therefore, the researcher can assume that the tourists approached were motivated to shop in those venues and for those particular products and services. This type of studies may then not be very useful in reflecting which segments among visitors to a destination are in fact interested in shopping, what motivates them to shop, what venues they prefer, what they like to buy and what other activities are they interested in at the destination. This current study was designed to shed light on these areas of research interests which have so far been relatively neglected.

Besides the scholarly contribution, the ultimate goal of this study is the applied understanding of tourists' motivation to shop while visiting the destination. The study concluded that, while Europeans represent the largest group of tourists in all five clusters, they are particularly well-represented among those tourists who tend to shop for the cultural experience or for the total shopping experience and prefer bazaars and arts and crafts stores. Following Tosun et al. (2007), the researcher proposes that, on the European market, bazaars and arts and crafts centres in Istanbul be promoted as places where tourists can experience local culture rather than places where they can buy things.

Although much smaller than the other groups, product-focused shoppers, are also worth pursuing by marketers for their dedication to shopping and, as previous studies have pointed out (Egresi, Arslan, 2016), for the large amount of money they are willing to spend on shopping. In this case, promotional strategies should focus especially on the Middle East and Africa and should emphasize the shopping opportunities waiting for tourists in Istanbul's malls.

Of the five groups resulted following market segmentation by motivation to shop, the least worth pursuing would be the 'reluctant shoppers' because many tourists in this group lack any interest in shopping. Therefore, any marketing strategies designed to persuade these tourists to shop and visit may be not only difficult to create and implement but also expensive and ineffective.

\section{Limitations}

One limitation is that, similar to most previous studies, this research surveyed only English - or Turkish speaking tourists in only one city. The study also excluded domestic tourists as these were more difficult to spot. All these choices that the author made may have influenced the outcomes of the study.

While the limitations outlined here may somewhat prevent generalization of the findings, they also present a great opportunity for future improvements. One possible new direction of research could be to replicate this market segmentation exercise in a number of tourist areas in Turkey and compare the resulting segmentation models. These areas could be selected based on different demographics (including country of origin) and travel behaviour. Another idea would be to apply this survey also to domestic tourists. While the Turkish tourism sector relies heavily on international tourists, the rising number of domestic tourists should not be overlooked. 


\section{Acknowledgements}

This study was conducted while the author was affiliated with Fatih University, Istanbul, Turkey and was made possible by a research grant provided by the university (number: P51061201_Y). The author would also like to thank the following students for providing assistance with the questionnaires (in alphabetical order): Cetin Duran, Tugba Esen, Betul Karakas, Hacer Sahin, Duygu Polat and Halil Yildirim.

\section{References}

Anderson, L.F., Littrell, M.A. 1995. Souvenir-purchase behaviour of women tourists. Annals of Tourism Research 22, 328-348. doi: 10.1016/0160-7383(94)ooo8o-8

Andreu, L., Kozak, M., Avci, N., Cifter, N. 2005.Market segmentation by motivation to travel: British tourists visiting Turkey. Journal of Travel and Tourism Marketing 19, 1-14. doi: 10.1300/Jo73v19no1_o1

Arnold, M., Reynolds, K. 2003. Hedonic shopping motivations. Journal of Retailing and Consumer Services 79, 77-95. doi: 10.1016/Soo22-4359(03)oooo7-1

Bansal, H., Eiselt, H.A. 2004. Exploratory research of tourist motivations and planning. Tourism Management 25, 387-396. doi: 10.1016/So261-5177(03)oo135-3

Bergadaa, M., Faure, C., Perrien, J. 1995. Enduring involvement with shopping. Journal of Social Psychology 135-1, 17-23. doi: 10.1080/oo224545.1995.9711396

Bieger, T., Laesser, C. 2002. Market segmentation by motivation: The case of Switzerland. Journal of Travel Research 41, 68-76. doi: 10.1177/004728750204100110

Boksberger, P.E. , Laesser, C. 2009. Segmentation of the senior travel market by the means of travel motivations. Journal of Vacation Marketing 15, 311-322. doi: 10.1177/1356766709335829

Brida, J.G., Disegna, M., Osti, L. 2012. Segmenting visitors of cultural events by motivation: A sequential non-linear clustering analysis of Italian Christmas Market visitors. Expert Systems with Applications 39, 11349-11356. doi: 10.1016/j.eswa.2012.03.041

Butler, R.W. 1991. West Edmonton Mall as a tourist attraction. The Canadian Geographer 35, 287-295.

Carmichael, B.A., Smith, W.W. 2004. Canadian domestic travel behaviour: A market segmentation study of rural shoppers. Journal of Vacation Marketing 10, 333-347. doi: 10.1177/135676670401000405

Chang, J., Chiang, C.H. 2006. Segmenting American and Japanese tourists on novelty-seeking at night markets in Taiwan. Asia Pacific Journal of Tourism Research 11, 291-406. doi: 10.1080/10941660600931242

Chen, C.-C., Wang, Y.-C., Lin, Y.-H., Jiang, J. 2014. Segmenting Taiwanese tourists to China by ethnic identity and generation. Journal of Vacation Marketing 20, 181-191.doi: 10.1177/1356766713507514

Chen, R.J.C. 2013. How can stores sustain their businesses? From shopping behaviours and motivations to environment preferences. Sustainability 5, 617-628. doi: 10.339o/su5020617

Choi, M.J., Heo, C.Y., Law, R. 2016. Progress in shopping tourism.Journal of Travel and Tourism Marketing 33, 1-24. doi: 10.1080/10548408.2014.969393

Chung, Y.-S., Wu, C.-L., Chiang, W.-E. 2013. Air passengers' shopping motivation and information seeking behaviour. Journal of Air Transport Management 27, 25-28. doi: 10.1016/j.jairtraman.2012.11.006

Crompton, J.L. 1979. Motivations for pleasure travel. Annals of Travel Research 24-2, 425-439.

Dann, G.M. 1981. Tourist motivation: An appraisal. Annals of Tourism Research 8, 187-224. doi: 10.1016/0160-7383(81)90082-7

Devesa, M., Laguna, M., Palacios, A. 2010. The role of motivation in visitor satisfaction: Empirical evidence in rural tourism. Tourism Management 31, 547-552. doi: 10.1016/j.tourman.2009.06.006

Diaz-Perez, F.M., Bethencourt-Cejas, M., AlvarezGonzalez, J.A. 2005. The segmentation of Canary Island tourism markets by expenditure: Implications for tourism policy. Tourism Management 26, 961-964. doi: 10.1016/j.tourman.2004.06.009

Dolnicar, S. 2004. Beyond 'commonsense segmentation': A systematics of segmentation approaches in tourism. Journal of Travel Research 42, 244-250. doi: 10.1177/0047287503258830

Dong, E., Wang, Y., Morais, D., Brooks, D. 2013. Segmenting the rural tourism market: The case of Potter County, Pennsylvania, USA. Journal of Vacation Marketing 19, 181-193. doi: 19.1177/1356766712471231

D’Urso, P., Disegna, M., Massari, R., Osti, L. 2016. Fuzzy segmentation of postmodern tourists. Tourism Management 55, 297-308. doi: 10.1016/j.tourman.2016.03.018

D’Urso, P., De Giovanni, L., Disegna, M., Massari, R. 2013. Bagged clustering and its application to tourism market segmentation. Expert Systems with Applications 40, 4944-4956. doi: 10.1016/j. eswa.2013.03.005

Eastlick, M.A., Feinberg, R.A. 1999. Shopping motives for mail catalog shopping. Journal of Business Research 45-3, 281-290.

Egresi, I. 2017. Tourists' satisfaction with shopping experience based on reviews on TripAdvisor. Tourism: An International Interdisciplinary Journal 65-3, 330-345.

Egresi, I. 2015. Tourists' shopping satisfaction in Istanbul's traditional markets. Proceedings of the Geobalcanica International Scientific Conference, Skopje, Macedonia, 5-7 June, 291-298 pp. Available at: http://geobalcanica.org/proceeding/ 
Egresi, I., Arslan, S. 2016. Shopping and tourism in Turkey: The perfect combination. In: Egresi, I. [ed.], Alternative Tourism in Turkey: Role, Potential Development and Sustainability. Springer International, Cham, Switzerland, 211-228 pp.

Egresi, I., Polat, D. 2016. Assessing tourists' satisfaction with their shopping experience in Istanbul. GeoJournal of Tourism and Geosites 9, 172-186. Available at: http://gtg.webhost.uoradea.ro/

Egresi, I., Kara, F. 2015. Predictors of tourists' shopping propensity: a case from Istanbul. Geographia Technica 102, 9-40. Available at: http://technicalgeography.org/

Fodness, D. 1994. Measuring tourist motivation. Annals of Tourism Research 21-3, 555-581.

Frochot, I., Morrison, A.M. 200o. Benefit segmentation: a review of its application to travel and tourism research. Journal of Travel and Tourism Marketing 9-4, 21-45. doi: 10.1300/Jo73vo9no4_02

Guiry, M., Magi, A., Lutz, R. 2006. Defining and measuring recreational shopper identity. Journal of the Academy of Marketing Science, 34-1, 74-83. doi: 10.1177/0092070305282042

Henderson, J.C., Chee, L., Mun, C.N., Lee, C. 2011. Shopping, tourism, and retailing in Singapore. Managing Leisure 16-1, 36-48. doi: 10.1080/13606719.2011.532599

Heung, V.C.S., Qu, J. 1998. Tourism shopping and its contribution to Hong Kong. Tourism Management 19, 383-386.doi: 10.1016/So261-5177(98)0oo29-6

Hobson, J.S.P., Christensen, M. 2001.Cultural and structural issues affecting Japanese tourists' shopping behaviour. Asia Pacific Journal of Tourism Research 6, 37-45.doi: 10.1080/10941660108722086

Hsieh, A-T., Chang, J. 2006. Shopping and tourist night markets in Taiwan. Tourism Management 27, 138-145. doi: 10.1016/j.tourman.2004.06.017

$\mathrm{Hu}, \mathrm{B}$., Yu, H. 2007. Segmentation by craft selection criteria and shopping involvement. Tourism Management 28, 1079-1092. doi: 10.1016/j.tourman.2006.07.006

Huang, S., Hsu, H.C. 2005. Mainland Chinese residents' perceptions and motivations of visiting Hong Kong: evidence from focus group interviews. Asia Pacific Journal of Tourism Research 10, 191-205. doi: 10.1080/10941660500135977

Hudson, S. 2000. The segmentation of potential tourists: constraint differences between men and women. Journal of Travel Research 38, 363-368. doi: 10.1177/004728750003800404

Iso-Ahola, S. 1990. Understanding leisure and recreation. Mapping the past, charting the future. In: Jackson, E.L. and Burton, T.L. [eds.], Motivation for Leisure. Vent, State College, PA, 247-279 pp.

Iso-Ahola, S. 1982. Towards a social psychology theory of tourism motivation: A rejoinder. Annals of Tourism Research 9, 256-262. doi: 10.1016/01067383(82)90049-4

Iso-Ahola, S. 1980. The Social Psychology of Leisure Recreation. Brown, Dubuque, IA, 436 pp.

Jansen-Verbeke, M. 1998. The synergism between shopping and tourism. In: Theobald, W.F. [ed.] Global Tourism, $2^{\text {nd }}$ edition, Butterworth-Heinemann, Oxford, 428-446 pp.

Jansen-Verbeke, M. 199o. Leisure + shopping = tourism product mix. In: Ashworth, G., Goodall, B. [eds.], Marketing Tourism Places, Routledge, London, 128-137 pp.

Jonsson, C., Devonish, D. 2008. Does nationality, gender and age affect travel motivation? A case of visitors to the Caribbean Island of Barbados. Journal of Travel and Tourism Marketing 25, 398-408. doi: 10.1080/10548400802508499

Keown, C. F. 1989. A model of tourists' propensity to buy: A case of Japanese visitors to Hawaii. Journal of Travel Research 27-3, 31-34.

Kim, J.H., Ritchie, B.W. 2012. Motivation-based typology: An empirical study of golf tourists. Journal of Hospitality and Tourism Research 36, 251-290. doi: 10.1177/1096348010388661

Kinley, T.R., Forney, J.A., Kim, Y.-K. 2012. Travel motivation as a determinant of shopping venue. International Journal of Culture, Tourism, and Hospitality Research, 6, 250-265.doi: 10:1108/17506181211246429

Kozak, M. 2002.Comparative analysis of tourist motivations by nationality and destinations. Tourism Management 23, 221-232. doi: 10.1016/S2615177(01)00090-5

LeHew, M.L.A., Wesley, S.C. 2007. Tourist shoppers' satisfaction with regional shopping mall experiences. International Journal of Culture, Tourism and Hospitality Research 1, 82-96. doi: 10.1108/17506180710729628

Littrell, M.A., Paige, R.C., Song, K. 2004. Senior travellers: tourism activities and shopping behaviours. Journal of Vacation Marketing 10, 348-362. doi: 10.1177/135676670401000406

Lopez-Guzman, T., Vieira-Rodriguez, A., RodriguezGarcia, J. 2014. Profile and motivations of European tourists on the Sherry wine route of Spain. Tourism Management Perspectives 11, 63-68. doi: 10.1016/j.tmp.2014.04.003

Lyu, S.O., Noh, E.J. 2016. Shopping decisions of international tourists to Korea: The Heckman sample selection approach. Journal of Destination Marketing and Management 6-4, 436-443. Available at: http://dx.doi.org/10.1016/j.jdmm.2016.07.001

Mak, B.F., Tsang, N.K., Cheung, I.C. 1999. Taiwanese tourists' shopping preference. Journal of Vacation Marketing 5, 190-198. doi: 10.1177/13576679900500207 
Maslow, A.H. 1943. A theory of human motivation. Psychological Review 50, 370-396. http://psychclassics.yorku.ca/Maslow/motivation.html

Mazanec, J. 1992. Classifying tourists into market segments: A neural network approach. Journal of Travel and Tourism Marketing 1-1, 39-60. doi: 10.1300/ Jo73vo1no1_04

Middleton, V.T.C., Clarke, J. 20o1. Marketing in Travel and Tourism ( $3^{\text {rd }}$ Edition). Butterworth-Heinemann, Woburn, MA, $487 \mathrm{pp}$.

Mody, M., Day, J., Sydnor, S., Jaffe, W., Lehto, X. 2014. The different shades of responsibility: Examining domestic and international travellers' motivations for responsible tourism in India. Tourism Management Perspectives 12, 113-124. doi: 10.1016/j.tmp.2014.09.008

Mok, C., Iverson, T.J. 200o. Expenditure-based segmentation: Taiwanese tourists to Guam. Tourism Management 21, 299-305. doi: 10.1016/So2615177(99)0006o-6

Moscardo, G. 2004. Shopping as a destination attraction: An empirical examination of the role of shopping in tourists' destination choice and experience. Journal of Vacation Marketing 10, 294-307. doi: 10.1177/135676670401000402

Murphy, L., Moscardo, G., Benckendorf, P., Pearce, P. 2011. Evaluating tourist satisfaction with the retail experience in a typical tourist shopping village. Retailing and Consumer Services 18, 302-310. doi: 10.1016/j.jretconser.2011.02.004

Oh, J.Y.J., Cheng, C.K., Lehto, X.Y., O’Leary, J.T. 2004. Predictors of tourists' shopping behaviour: examinations of socio-demographics characteristics and trip typologies. Journal of Vacation Marketing 10, 308-319. doi: 10.1177/135676670401000403

Park, D.- B., Yoon, Y.- S. 2009. Segmentation by motivation in rural tourism: A Korean case study. Tourism Management 30, 99-108.doi: 10.1016/j.tourman.2008.03.011

Park, K.-S., Reisinger, Y., Noh, E.-H. 2010. Luxury shopping in tourism. International Journal of Tourism Research 12, 164-178. doi: 10.1002/jtr.744

Park, K.-S., Reisinger, Y., Kang, H.-J. 2008. Visitors' motivation for attending the South Beach Wine and Food Festival, Miami Beach, Florida. Journal of Travel and Tourism Marketing 25, 161-181. doi: 10.080/10548400802402883

Pesonen, J., Laukkanen, T., Komppula, R. 2011. Benefit segmentation of potential wellbeing tourists. Journal of Vacation Marketing 17-4, 303-314. doi: 10.1177/1356766711423322

Peter, S., Anandkumar, V. 2011. A study on the sources of competitive advantage of Dubai as a shopping tourism destination. Proceedings of the International Business Research Conference. Available at: http://www.wbiconpro.com/510-Sangeeta.pdf
Pizam, A., Jansen-Verbeke, M., Steel, L. 1997. Are all hospitality tourists alike regardless of nationality? Journal of International Hospitality, Leisure \& Tourism Management 1, 19-39. doi: 10:1300/J268vo1no1_03 Rid, W., Ezenduji, I.O., Probstl-Haider, U. 2014. Segmentation by motivation for rural tourism activities in The Gambia. Tourism Management 40, 102116. doi: 10.1016/j.tourman.2013.05.006

Rosenbaum, M.S., Spears, D.L. 2006. An exploration of spending behaviours among Japanese tourists. Journal of Travel Research 44, 467-473. doi: 10.1177/0047287505282949

Swanson, K.K., Horridge, P.E. 2006. Travel motivations as souvenir purchase indicators. Tourism Management 27, 671-683. doi: 10:1016/j.tourman.2005.03.001

Tkaczynski, A., Rundle-Thiele, S.R., Beaumont, N. 2009. Segmentation: A tourism stakeholder view. Tourism Management 30, 169-175. doi: 10.1016/j. tourman.2008.05.010

Timothy, D.J. 2005.Shopping Tourism, Retailing and Leisure. Channel View Publications, Clevedon, 235 pp.

Timothy, D.J.,Butler, R.W. 1995. Cross-border shopping: A North-American perspective. Annals of Tourism Research 22-1, 16-34.

Tosun, C., Temizkan, S.P., Timothy, D.J. Fyall, A. 2007. Tourist shopping experiences and satisfaction. International Journal of Tourism Research 9, 87-102. doi: $10.1002 /$ jtr.595

Weaver, P.A., McCleary, K.W., Lepisto, L., Damonte, L.T. 1994. The relationship of destination selection attributes to psychological, behavioural and demographic variables. Journal of Hospitality and Leisure Marketing 2-2, 93-109. Available at: http://www. tandfonline.com/toc/whmm19/2/2

$\mathrm{Xu}, \mathrm{J}$., Chan, S. 2016. A new nature-based motivation model: Testing the moderating effects of the push motivation. Tourism Management Perspectives 18, 107-110. doi: 10.1016/j.tmp.2016.01.001

Yoon, Y., Uysal, M. 2005. An examination of the effects of motivation and satisfaction on destination loyalty: A structural model. Tourism Management 26, 45-56. doi: 10.1016/j.tourman.2003.08.016

Yuksel, A. 2007. Tourist shopping habitat: effects on emotions, shopping value and behaviours. Tourism Management 28, 58-69. doi: 10.1016/j.tourman.2005.07.017

Yuksel, A. 2004. Shopping experience evaluation: a case of domestic and international visitors. Tourism Management 25, 751-759. doi: 10.1016/j.tourman.2003.09.012

Yuksel, A., Yuksel, F. 2002. Market segmentation based on tourists' dining preferences. Journal of Hospitality and Tourism Research 26, 315-331. doi: 10.1177/109634802237482 